\title{
CONSIDERACIÓN EXPLÍCITA DEL DAÑO ACUMULADO EN EL DISEÑO SISMICO DE ESTRUCTURAS A TRAVÉS DE FACTORES DE REDUCCIÓN DE RESISTENCIA POR DUCTILIDAD
}

\author{
Edén Bojórquez Mora ${ }^{(1,2)}$, Amador Terán Gilmore ${ }^{(3)}$, \\ Juan Bojórquez Mora ${ }^{(1)}$ y Sonia E. Ruiz Gómez ${ }^{(4)}$
}

\begin{abstract}
RESUMEN
Una estructura que incurre en varios ciclos de comportamiento plástico cuando se somete a una excitación sísmica severa puede sufrir una degradación excesiva de sus propiedades estructurales, lo que puede provocar su falla a niveles de deformación que están muy por debajo de los que alcanza cuando se le sujeta a un estado de desplazamiento monótonamente creciente. Debido a esto, es necesario incorporar al diseño sísmico información que permita caracterizar numéricamente la severidad de las demandas plásticas acumuladas. Una manera práctica de lograr esto consiste en plantear factores de reducción de resistencia por ductilidad que tomen en cuenta el efecto de las demandas acumuladas a través de definir una ductilidad reducida. En este trabajo, el efecto del daño acumulado se incorpora a la definición de factores de reducción de resistencia a través del índice de daño de Park y Ang. Para ello, se estiman las ductilidades máximas que según dicho índice pueden acomodar sistemas de un grado de libertad sujetos a movimientos del terreno registrados en distintas zonas del Valle de México, y se determinan factores de reducción de resistencia por ductilidad que consideran la acumulación del daño a través de dichas ductilidades. Se discuten los resultados obtenidos y se hacen recomendaciones de como incorporar los efectos de las demandas acumuladas de deformación plástica al diseño sísmico de estructuras.
\end{abstract}

\begin{abstract}
A structure that undergoes several cycles of plastic behavior when subjected to a severe seismic excitation can exhibit excessive degradation of its structural properties, and as a consequence failure at deformation levels that are significantly smaller than the one it can develop when subjected to a state of monotonically increasing deformation. Because of this, it is necessary to incorporate into seismic design information that allows for a numerical characterization of the severity of cumulative plastic deformation demands. One practical way to achieve this is to formulate strength reduction factors that take into account the effect of cumulative demands through the definition of a reduced ductility. In this paper, the effect of cumulative damage is incorporated into the definition
\end{abstract}

Artículo recibido el 20 de marzo de 2008 y aprobado para su publicación el 3 de marzo de 2009. Se aceptarán comentarios y/o discusiones hasta cinco meses después de su publicación

\footnotetext{
(1) Facultad de Ingeniería, Universidad Autónoma de Sinaloa, Calzada de las Américas y Boulevard Universitarios S/N, Ciudad Universitaria,Culiacán, Sinaloa, México, C.P.80040.ebojorq@uas.uasnet.mx bojorquezeden.mora@unina.it

(2) , Dipartimento di Ingegneria Strutturale, Università degli Studi di Napoli Federico II, Via Claudio 21, 80125 Napoli Italia

(3) Universidad Autónoma Metropolitana, Depto. de Materiales, Av. San Pablo 180, Col. Reynosa Tamaulipas, 02200 México, D.F.tga@correo.azc.uam.mx

(4) Instituto de Ingeniería, Universidad Nacional Autónoma de México, Coyoacán, C.P. 04510, México, D.F. sruizg@iingen.unam.mx
} 
of strength reduction factors through the use of the Park and Ang damage index. For this purpose, the maximum ductilities that according to this index can be accommodated by single-degree-offreedom systems located in different zones of the Valley of Mexico are estimated, and strength reduction factors are determined as a function of these ductilities. After discussing the numerical results that are obtained, recommendations are made on how to incorporate the effect of cumulative plastic deformation demands into seismic design.

\section{INTRODUCCIÓN}

Una estructura que se somete a varios ciclos de comportamiento plástico puede sufrir un deterioro importante en sus características mecánicas, lo que puede resultar en que falle a un nivel de ductilidad o desplazamiento significativamente menor al que se supone durante su diseño. Debido a esto, en algunos casos, como lo es el de las estructuras sujetas a la acción de excitaciones sísmicas de larga duración, las demandas máximas de deformación no permiten caracterizar adecuadamente el nivel esperado de daño estructural (Reinoso 1996, Bojórquez 2007, Rodríguez y Padilla 2008). Para explicar esto, puede plantearse el caso de dos estructuras iguales que alcancen la misma demanda máxima de desplazamiento durante excitaciones sísmicas con muy diferente duración (corta versus larga). Es de esperar que aquella estructura sujeta al sismo de larga duración exhiba durante su respuesta sísmica un número considerable de ciclos de comportamiento plástico, cuyo efecto acumulado en términos de degradación y daño por fatiga sea mucho mayor que aquel que corresponde a la estructura sujeta al sismo de corta duración. Casos como el de la estructura sujeta a la excitación de larga duración solo pueden ser correctamente caracterizados si se considera explícitamente, además de la demanda máxima de desplazamiento, otros parámetros que contemplen el efecto de las demandas acumuladas de deformación plástica (Fajfar 1992, Ballio y Castiglioni 1994, Manfredi 2001, Malhotra 2002, Chou y Uang 2003, Boomer et al. 2004, Chai 2005, Iervolino et al. 2006, Bojórquez et al. 2006, Hancock y Bommer 2006, Bojórquez et al. 2008a y 2008b). En el caso de México, las demandas acumuladas son particularmente dañinas para edificios desplantados en la Zona del Lago del Distrito Federal y cuyos periodos fundamentales de vibración estén cercanos al periodo dominante del terreno (Terán-Gilmore 2001, Bojórquez y Ruiz 2004).

Para considerar la acumulación de las demandas plásticas en las estructuras sismo-resistentes, y con ello la influencia de la duración sobre su respuesta sísmica, se han desarrollado diversos indicadores o índices de daño. Algunos de estos índices resultan de la combinación lineal de las demandas de energía histerética normalizada y del desplazamiento máximo (Park y Ang 1985, Bozorgnia y Bertero 2001); de la energía histerética normalizada respecto a la máxima demanda elástica (Rodríguez 1997); o de la consideración explícita del número y amplitud de los ciclos de comportamiento plástico a través del índice de acumulación lineal del daño (Krawinkler y Zohrei 1983). Aunque se ha propuesto el uso de diversas metodologías de diseño que utilizan de manera directa índices de daño, lo cierto es que aún es necesario refinar tales procedimientos e índices. Por el momento parecería que la manera más factible de incorporar el daño acumulado al diseño sísmico es a través del concepto de ductilidad objetivo (Fajfar 1992). El uso de dicho concepto permite el planteamiento de factores de reducción de resistencia que incorporen de manera explícita el daño acumulado (Arroyo y Terán 2002, Bojórquez y Ruiz 2004, Teran-Gilmore y Jirsa 2007). En otras palabras, los efectos nocivos del daño acumulado pueden controlarse a través de proporcionarle a la estructura una resistencia lateral tal que controle su demanda máxima de ductilidad durante la excitación sísmica dentro del umbral definido por la ductilidad objetivo.

El primer objetivo del presente estudio es evaluar, bajo consideración explícita del efecto del las demandas acumuladas de deformación plástica, la ductilidad objetivo en sistemas de un grado de libertad que representan estructuras sismo-resistentes desplantadas en distintas zonas del Valle de México. Para 
ello, se utiliza el índice de daño de Park y Ang (1985). Un segundo objetivo es recomendar sobre la conveniencia de incorporar en determinados casos el efecto de las demandas acumuladas de deformación plástica durante el diseño sismo-resistente, e identificar aquellos otros en los que la consideración exclusiva de la demanda máxima de desplazamiento puede dar lugar a un diseño adecuado.

\section{ALGUNAS LIMITACIONES DE LA DEMANDA MÁXIMA COMO PARÁMETRO DE DISEÑO}

Los requerimientos normativos de diseño sismo-resistente suelen basarse en el uso de espectros elásticos de diseño que se reducen de manera independiente por ductilidad y sobre-resistencia. Mientras que en algunos casos estas reducciones son explícitas, varios formatos las consideran de manera implícita. Por ejemplo, la versión 2004 de las Normas Técnicas Complementarias para Diseño por Sismo (NTCDS) del Reglamento de Construcciones para el Distrito Federal (RCDF), hacen consideración explícita, tanto en su cuerpo principal como en su Apéndice A, de la reducción por ductilidad del espectro elástico de resistencia. En cuanto a la sobre-resistencia, mientras que el cuerpo principal de las NTCDS la considera de manera implícita a través de presentar un espectro elástico con ordenadas reducidas, el Apéndice A la toma en cuenta de manera explícita a través de un factor de reducción asociado a sobre-resistencia. Es interesante notar, como lo han hecho autores como Whittaker et al. (1999), que las reducciones por ductilidad y sobre-resistencia pueden considerarse y plantearse como independientes, de tal manera que cada una de estas reducciones puede estudiarse y calibrarse por separado. Aunque este artículo hace algunas referencias al tema de sobre-resistencia, el material que se presenta está orientado a discutir el planteamiento de factores de reducción por ductilidad que caractericen adecuadamente las demandas máximas y acumuladas de comportamiento plástico que se esperan en las estructuras ubicadas en las diferentes zonas sísmicas del Valle de México.

Los requerimientos normativos usados para el diseño de resistencia de estructuras ubicadas en el Distrito Federal han sido derivados a partir de un criterio de control de deformación plástica máxima (Ordaz et al. 2003); y es necesario, por lo planteado hasta el momento, estudiar bajo qué circunstancias el control exclusivo del desplazamiento máximo garantiza o no el buen desempeño de estas estructuras. Para ilustrar lo anterior, considere por un lado el movimiento del terreno registrado en la dirección este-oeste de la estación SCT durante el sismo ocurrido el 19 de septiembre de 1985 (figura 1). Por otro lado, considere el movimiento que se obtiene al tomar una pequeña porción de la fase intensa del registro SCT (parte en línea gruesa de la figura 1 que se delimita con las líneas verticales discontinuas: "SCT recortado").

En la figura 2 se comparan para ambos registros los espectros lineales, y algunos no lineales obtenidos a partir de un modelo elasto-plástico perfecto. Se observa que tanto el espectro lineal como el espectro no lineal correspondiente a ductilidad de dos para el registro SCT pueden obtenerse por medio de considerar solo una pequeña porción del registro. Lo anterior implica que se obtendría un diseño similar al utilizar cualquiera de los dos registros. Pero ¿qué pasa con el desempeño de la estructura? Para aportar una posible respuesta a esta pregunta, considere una estructura que se somete a la acción de ambos registros. Dicha estructura está representada por un sistema de un grado de libertad (1GL) con las siguientes características: periodo de vibración $(T)$ de 1.5 segundos, coeficiente sísmico $\left(C_{y}\right)$ de 0.2 , porcentaje de amortiguamiento crítico $(\xi)$ de $5 \%$, comportamiento elasto-plástico perfecto, y una degradación de resistencia lateral basada en la ley constitutiva de energía histerética planteada por Bojórquez y Rivera (2005). La respuesta fuerza-desplazamiento de la estructura ante la acción de ambos registros se ilustra en la figura 3. En primer lugar, se observa cómo el máximo desplazamiento para ambos casos es prácticamente igual, lo que implica que en términos de desplazamiento máximo, ya sea elástico o inelástico, puede considerarse un mismo nivel de respuesta. En el caso del número de ciclos de comportamiento plástico se observan diferencias importantes. Como era de esperarse, cuando se utiliza el 
registro completo se demanda a la estructura un mayor número de ciclos, lo que tiene como consecuencia un mayor daño estructural y una mayor reducción en la resistencia debido al efecto acumulado de degradación. Es evidente que en algunos casos, utilizar únicamente el desplazamiento máximo para fomentar el buen desempeño estructural puede resultar en diseños del lado de la inseguridad, y que en estos casos será necesario utilizar parámetros que consideren el efecto del daño acumulado, como pueden ser el tiempo de duración del movimiento, la energía histerética disipada (representada por el área encerrada en los ciclos de comportamiento plástico) o algún indicador de daño que tome en cuenta la acumulación de demandas plásticas.

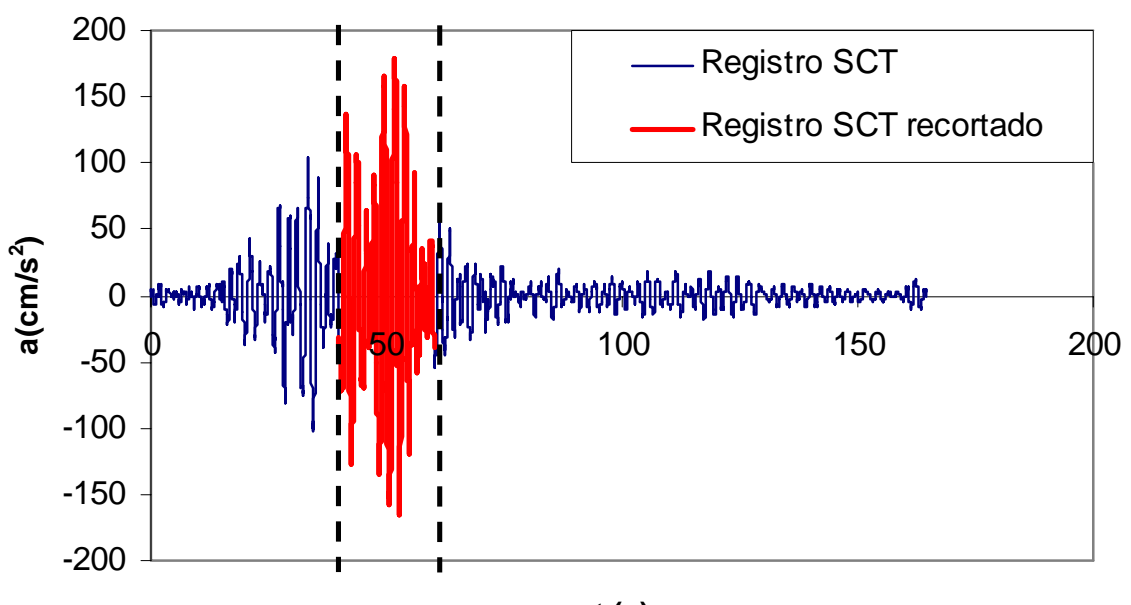

$t(s)$

Figura 1. Registro sísmico SCT (sismo del 19 de septiembre de 1985).

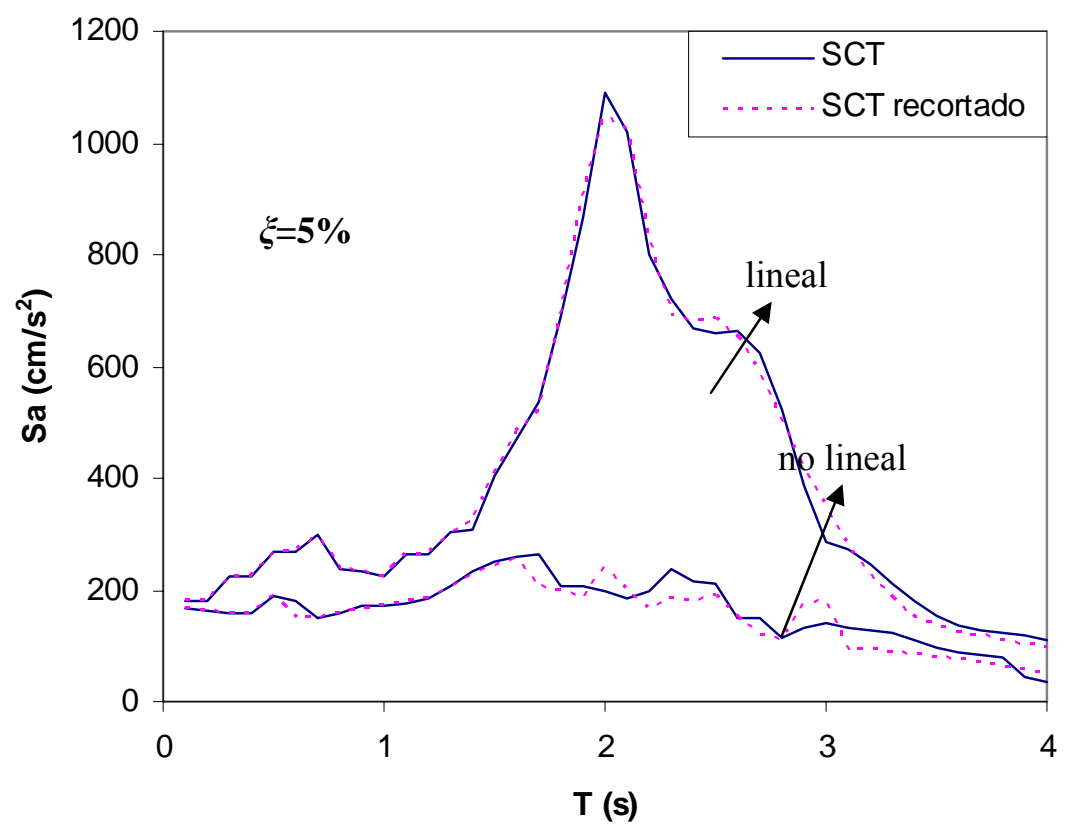

Figura 2. Espectros de pseudo-aceleración correspondientes al registro SCT. 


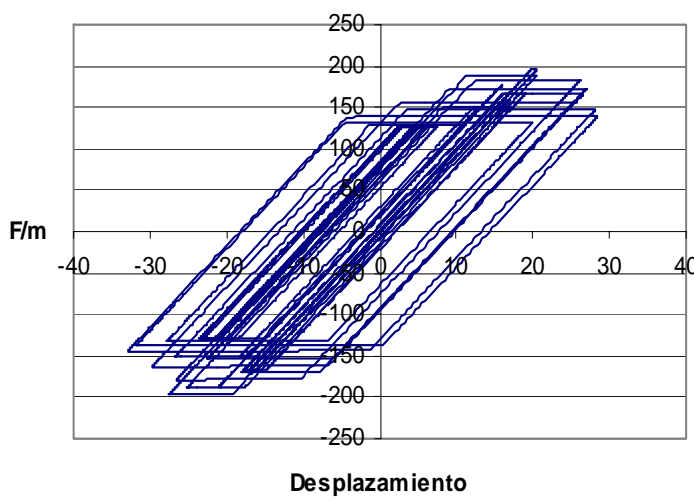

a) Completo

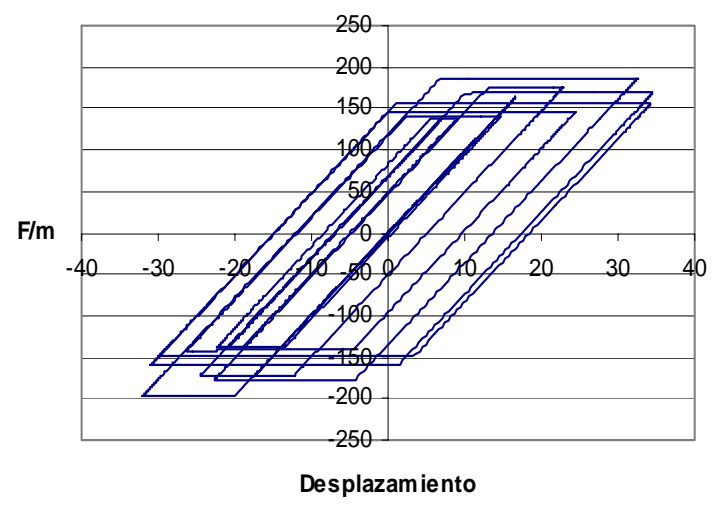

b) Recortado

Figura 3. Respuesta de sistema de $1 \mathrm{GL}$ ante registro SCT.

\section{ÍNDICES DE DAÑO}

La selección de un índice de daño para llevar a cabo un estudio analítico que resulte en recomendaciones de diseño debe hacerse cuidadosamente. Por un lado, todo índice de daño tiene limitaciones, de tal manera que los resultados que se obtengan a partir de su uso deben interpretarse cuidadosamente. Esto limita en ocasiones el alcance de las conclusiones que puedan obtenerse, y plantea problemas en cuanto a la selección del índice adecuado. Por el otro lado, es importante mencionar que el uso de diversos índices de daño, planteados a partir de simplificaciones y suposiciones muy diferentes, resulta en demandas de resistencia muy similares cuando se consideran las demandas acumuladas de deformación plástica (Terán y Jirsa 2005). Esto reduce las implicaciones de seleccionar un índice de daño en particular, y de alguna manera, aporta certeza a las tendencias observadas y a las sugerencias de diseño que se hagan. En este artículo se utiliza el índice de Park y Ang por ser el mejor sustentado en términos de calibración experimental y de campo (Park and Ang 1985, Stephens and Yao 1987, Williams and Sexsmith 1997, Silva and López 2001), y porque se le considera una referencia obligada entre los investigadores que estudian los efectos de las demandas acumuladas de deformación plástica.

\section{Índice de daño de Park y Ang}

Acorde a Park y Ang (1985), el nivel de daño estructural en elementos y estructuras de concreto reforzado sujetas a cargas cíclicas puede estimarse a partir de la combinación lineal de las demandas máximas y acumuladas de deformación plástica:

$$
I_{D P A}=\frac{\mu_{m}}{\mu}+\beta \frac{E_{H}}{F_{y} d_{y} \mu}
$$

donde $\mu_{m}$ es la máxima ductilidad que desarrolla la estructura cuando se somete a la acción de un movimiento sísmico; $\mu$ la relación entre el desplazamiento último que puede alcanzar el sistema cuando se le sujeta a un estado de deformación monótonamente creciente y el desplazamiento de fluencia (ductilidad última); $\beta$ un parámetro que depende de las características estructurales y que caracteriza la estabilidad del ciclo histerético; $E_{H}$ la demanda de energía histerética; y finalmente, $F_{y}$ y $d_{y}$, la fuerza y desplazamiento de 
fluencia, respectivamente. En este trabajo se utiliza $\beta$ de 0.15 , que corresponde a estructuras con un detallado sísmico adecuado (Cosenza et al. 1993). Teóricamente, $I_{D P A}$ igual a cero representa que la estructura no ha sufrido daño, mientras que un valor igual a la unidad indica la falla del sistema.

Para el caso de falla ( $I_{D P A}=1$ ), la ductilidad objetivo o máxima permisible que puede desarrollar el sistema puede expresarse como (Fajfar 1992):

$$
\mu_{m}=\frac{\sqrt{1+4 \beta \gamma^{2} \mu}-1}{2 \beta \gamma^{2}}
$$

donde:

$$
\gamma=\frac{\sqrt{E_{H} / m}}{\omega d_{m}}
$$

En la ecuación 3, $m$ representa la masa del sistema, $d_{m}$ su desplazamiento máximo y $\omega$ su frecuencia circular. La ecuación 2 muestra que la ductilidad objetivo queda controlada por los parámetros $\beta$ y $\gamma$, y la ductilidad última del sistema $\mu$.

A pesar de ser ampliamente usado para estudiar el efecto de las demandas acumuladas de deformación plástica, el índice de Park y Ang exhibe limitaciones e inconsistencias derivadas de las suposiciones simplificadoras a partir de las cuales se planteó (Chai y Romstad 1997, Kunnath et al. 1997). Esto no debe considerarse como una desventaja particular de este índice, ya que como recalcan Williams y Sexsmith (1995) y Mehanny y Deierlein (2000), todo índice de daño exhibe este tipo de problemas.

Mucha de la incertidumbre involucrada en el uso de índices de daño surge de la alta variabilidad que exhibe la capacidad de deformación de las estructuras sismo-resistentes. Esto normalmente resulta en que se asigne a las estructuras capacidades de deformación máxima y acumulada que están por debajo de sus capacidades reales. Otra fuente de incertidumbre se centra alrededor de la definición de falla. Normalmente, la falla por fatiga indicada por un índice de daño no está asociada al colapso o falla absoluta de un elemento o sistema estructural, sino a un estado de utilidad delimitado por un nivel máximo de degradación (particularmente de resistencia) que no estamos dispuestos a sobrepasar durante una excitación sísmica. Esto es, se considera como falla el que la estructura haya alcanzado un estado de degradación tal que su capacidad sismo-resistente no se considere adecuada para resistir las demandas sísmicas esperadas. La definición de si la estructura tiene capacidad adecuada o no se basa en un juicio que tiene mucho de subjetivo.

Como se comentó antes, algunos de los problemas con los índices de daño son problemas de concepción asociados a una suposición simplificadora. En el caso del índice de Park y Ang, sus autores consideraron atractivo suponer que el nivel de daño puede estimarse a partir de una combinación lineal de las demandas máxima y acumulada de deformación. Esto resulta en una sobrevaluación del daño en casos en que estas variables exhiban una fuerte dependencia, como sucede cuando se sujeta a la estructura a un estado de desplazamiento monótonamente creciente. Para ilustrar lo anterior, considere un sistema o elemento estructural con comportamiento elasto-plástico perfecto y sujeto a un estado de desplazamiento monótonamente creciente. Para este caso, el índice de Park y Ang puede expresarse como: 


$$
I_{D P A}=\frac{\mu_{m}}{\mu}+\beta \frac{\left(\mu_{m}-1\right)}{\mu}
$$

Si se considera que un sistema exhibe daño nulo mientras su comportamiento permanece elástico, un sistema que alcance el límite de su intervalo lineal de comportamiento $\left(\mu_{m}=1\right)$ debe exhibir daño nulo. Sin embargo, la ecuación 4 resulta en un valor mayor que cero:

$$
I_{D P A}=\frac{1}{\mu}
$$

Puede concluirse que a medida que la capacidad de ductilidad última en la estructura se reduce, se incrementa el nivel de sobrevaluación del daño estructural. Esto puede apreciarse en la figura 4a, donde $S_{D l i}$ representa dicho nivel. Bajo una consideración similar, pero ahora para el caso de falla ante un estado de desplazamiento monótonamente creciente, la ecuación 4 resulta para $\beta$ de 0.15 en:

$$
I_{D P A}=1+0.15-\frac{0.15}{\mu}
$$

Para este caso, la diferencia entre 0.15 y $0.15 / \mu$ en la ecuación 6 representa el nivel de sobrevaluación. Esto se muestra en la figura $4 \mathrm{~b}$, donde $S_{D l s}$ representa el nivel de sobrevaluación. La figura 5 resume parte de los resultados presentados en la figuras 4. Los círculos de la figura 5 ubicados en las coordenadas $(0,0)$ y $(1,1)$ indican el caso ideal en que $I_{D P A}$ sería una representación perfecta de daño. Mientras que para la condición de falla del sistema, las mayores sobrevaluaciones ocurren para ductilidades grandes, lo contrario ocurre en el límite inferior, para el cual la estructura todavía exhibe comportamiento elástico. No deja de ser interesante mencionar que algunos investigadores han propuesto índices de daño basados en el de Park y Ang, y que corrigen las sobrevaluaciones ilustradas por medio de las figuras 4 y 5 (Bozorgnia y Bertero 2001).

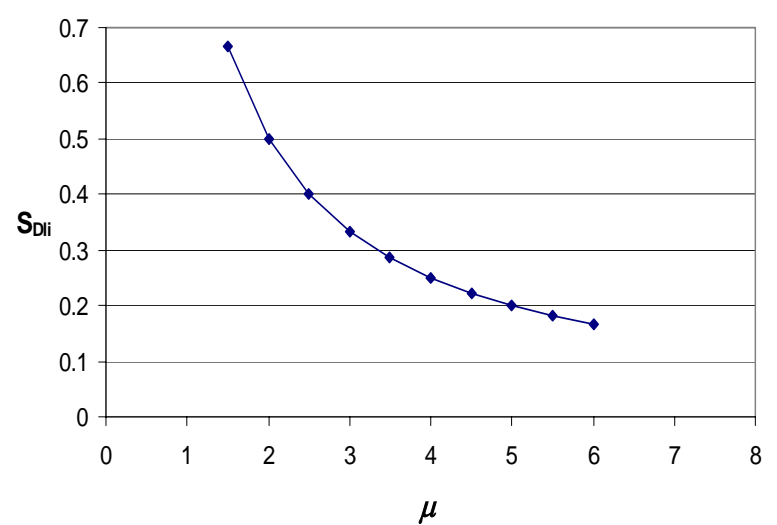

a) Nivel Elástico

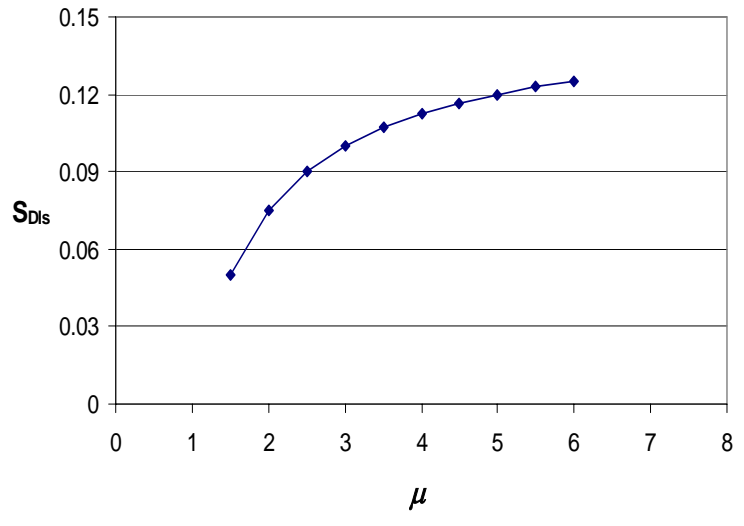

b) Falla debida a desplazamiento monótonamente creciente

Figura 4. Sobrevaluación de $I_{D P A}$ para representar el daño estructural. 
Otro tipo de problemas con el uso de índices de daño surge cuando estos se aplican a situaciones que no fueron contempladas durante su formulación. Esto puede ilustrarse a partir de la ligera sobrestimación que el índice de Park y Ang hace del nivel de daño en estructuras sismo-resistentes sujetas a altas demandas de energía. Conforme explican Terán y Jirsa (2005), esto se debe a que dicho índice no considera de forma explícita la manera en que la estructura acomoda las demandas de energía plástica, y por tanto a su inhabilidad de contemplar que la energía plástica que disipa hasta su falla un elemento o estructura puede cambiar sustancialmente en función de la amplitud de los ciclos plásticos.

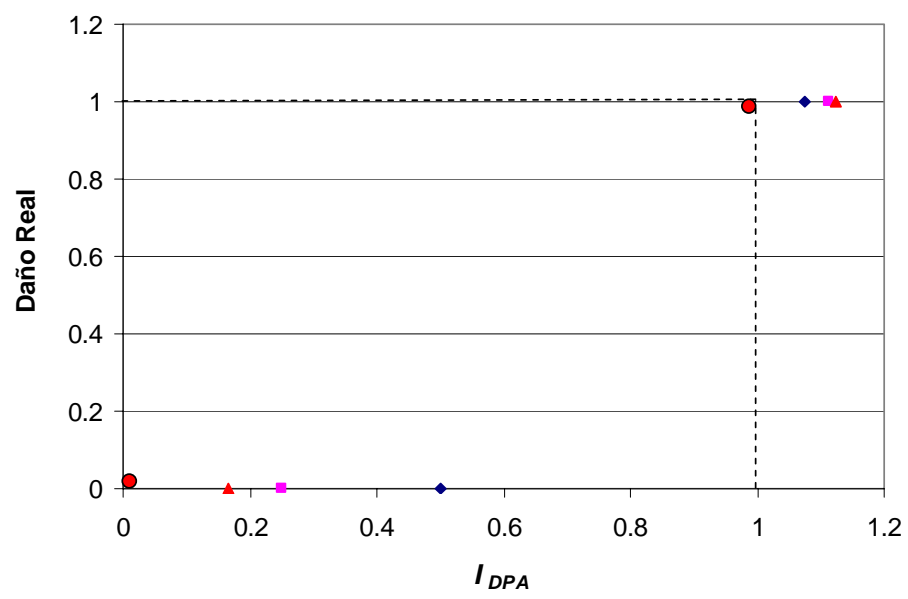

Figura 5. Comparación entre daño real e índice de Park y Ang.

Puede concluirse a partir de la discusión presentada en esta sección, que el uso del índice de Park y Ang tenderá a sobrevaluar el efecto de las demandas acumuladas de deformación plástica en dos casos extremos: A) Cuando las demandas de energía plástica tiendan a ser bajas, y B) Cuando las demandas de energía tiendan a ser muy altas. Esto junto con los valores conservadores que suelen asignarse a la capacidad que tiene la estructura para acomodar sus demandas plásticas resulta por lo general en estimaciones conservadoras del nivel de daño estructural o de las demandas de resistencia que requieren las estructuras para controlar dicho nivel de daño.

\section{Otros índices de daño}

Un índice de daño que puede tomar en cuenta el cambio de la capacidad de disipación de energía de una estructura en función de su historia de desplazamientos, puede ser formulado a partir de la teoría de acumulación lineal de daño (hipótesis de Miner). La hipótesis de Miner considera que el daño inducido por cada excursión plástica es independiente del daño producido por cualquier otra incursión, de tal manera que se requiere de una convención clara para definir y delimitar cada excursión (Powell y Allahabadi 1987, Terán y Jirsa 2005). Cosenza y Manfredi (1996) han formulado la hipótesis de Miner conforme a lo siguiente:

$$
I_{D M H}=\sum_{i=1}^{N e x c}\left(\frac{\delta_{p i}}{\delta_{u c p}}\right)^{b}
$$


donde Nexc es el número total de excursiones plásticas, $\delta_{u c p}$ el desplazamiento cíclico plástico último, $\delta_{p i}$ el desplazamiento plástico de la $i$-ésima excursión, y $b$ el parámetro estructural que caracteriza la estabilidad del ciclo histerético. Después de estudiar el trabajo experimental de varios investigadores en elementos de concreto reforzado y acero, Powell y Allahabadi (1987) sugieren que para fatiga de bajo número de ciclos, los valores típicos de $b$ oscilan entre 1.6 y 1.8. De hecho, se ha sugerido que un $b$ de 1.5 es un valor razonablemente conservador para ser utilizado en el diseño sísmico y en la evaluación de daño en estructuras de concreto y acero con un detallado sísmico adecuado (Krawinkler y Zohrei 1983, Baik et al. 1988, Cosenza y Manfredi 1996). $I_{D M H}$ igual a uno implica falla incipiente.

Terán y Jirsa (2005) han propuesto recientemente un modelo simple para evaluar la ocurrencia de fatiga de bajo número de ciclos. Este modelo representa una simplificación de la teoría de acumulación lineal de daño a través de la suposición de una distribución fija de excursiones plásticas en función de su amplitud:

$$
E_{H}=\frac{1.5}{(2-b)}(\mu-1) F_{y} \delta_{y}
$$

donde $b$ es el parámetro estructural que caracteriza la estabilidad del ciclo histerético. El valor de $E_{H}$ estimado acorde a la ecuación 8 establece la demanda de energía plástica que puede acomodar la estructura antes de fallar por fatiga de bajo número de ciclos. Para el diseño sísmico de estructuras de concreto y acero con un detallado sísmico adecuado, es razonable utilizar $b=1.5$ (Terán y Jirsa 2005).

\section{Comparación del uso de diferentes índices de daño}

El impacto del uso práctico de diferentes índices de daño puede evaluarse a través de comparar los resultados obtenidos a partir de su aplicación. Tanto Cosenza et al. (1993) como Terán y Jirsa (2005) han encontrado que las demandas de resistencia derivadas del índice de daño de Park y Ang son muy similares a las arrojados por el uso de otros índices de daño. En particular, Terán y Jirsa (2005) comparan las demandas de resistencia derivadas de los tres índices presentados en este artículo, y a partir de una discusión detallada de dicha comparación, concluyen que es mínimo el impacto de utilizar uno u otro modelo para establecer la resistencia lateral de diseño de una estructura cuando se consideran explícitamente las demandas acumuladas de deformación plástica. La figura 6 ilustra lo anterior para estructuras con comportamiento elasto-plástico perfecto y un detallado sísmico adecuado, y sujetas a excitaciones típicas de terreno firme y de la Zona del Lago del Distrito Federal (el letrero $I_{D T J}$ corresponde a los resultados obtenidos con el índice de Terán y Jirsa).

Si se considera que dentro del contexto de diseño sísmico actual el cortante basal de la estructura es la propiedad estructural que debe diseñarse, los resultados que muestra la figura 6 sugieren que el impacto de utilizar uno u otro modelo de daño es mínimo. Note que los requerimientos de resistencia obtenidos a partir de $I_{D P A}$ tienden a ser ligeramente menores que los obtenidos con $I_{D M H}$ e $I_{D T J}$ para estructuras sujetas a movimientos con muy alto contenido de energía y cuyo $T$ esta cercano al periodo dominante del terreno (que es igual a dos segundos en el caso de la figura $6 \mathrm{~b}$ ). Puede concluirse que en términos generales, las conclusiones que puedan obtenerse a partir de la aplicación de un índice de daño serán muy similares a las obtenidas a partir de otros. En este artículo se usa el índice de Park y Ang. 


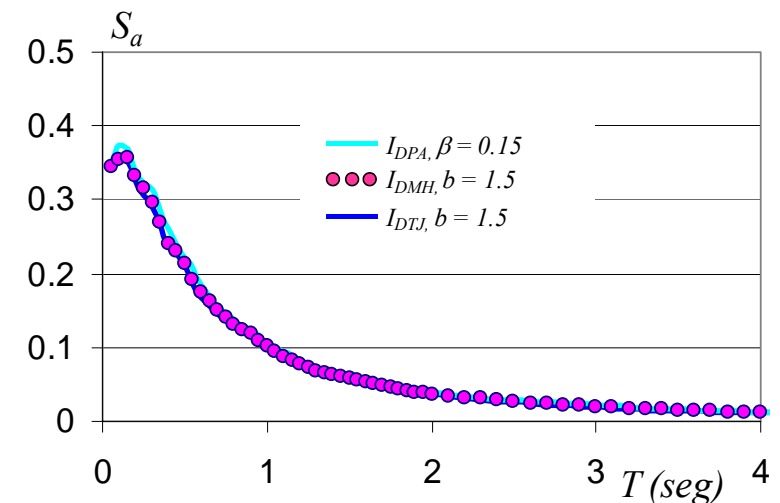

a) Suelo Firme, California

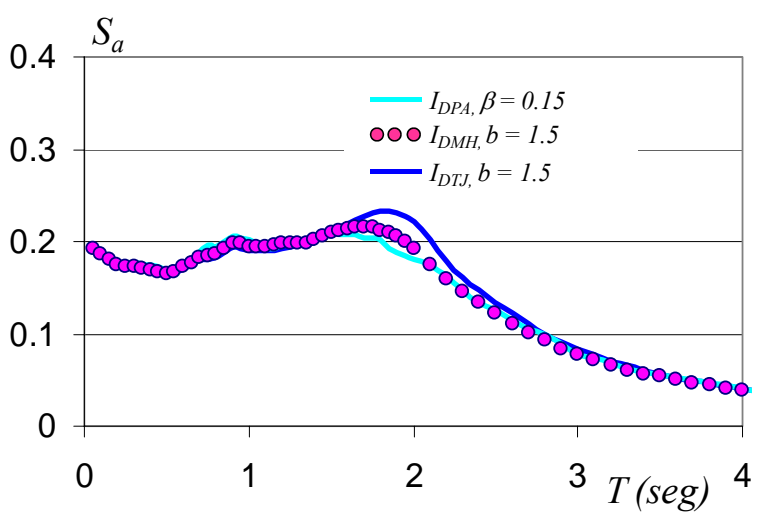

b) Suelo Blando, Distrito Federal

Figura 6. Resistencias laterales de diseño obtenidas a partir de tres modelos de daño, $\mu=5$.

\section{EVALUACIÓN DE LA DUCTILIDAD OBJETIVO PARA EL VALLE DE MÉXICO}

Para la evaluación de la ductilidad objetivo se utilizaron un total de 120 movimientos sísmicos registrados en distintas zonas del Valle de México durante eventos sísmicos de subducción (las zonas se

establecieron conforme a lo indicado por el cuerpo principal de las NTCDS). Se seleccionaron los eventos resumidos en la tabla 1, que se caracterizan por tener magnitudes mayores o iguales a 6.9.

Tabla 1. Características de los eventos sísmicos utilizados

\begin{tabular}{cccccc}
\hline Sismo & Fecha & Magnitud & $\begin{array}{c}\text { Hr:Min:Sec } \\
(\mathrm{GMT})\end{array}$ & $\begin{array}{c}\text { Latitud } \\
(\mathrm{N})\end{array}$ & $\begin{array}{c}\text { Longitud } \\
(\mathrm{E})\end{array}$ \\
\hline 1 & $68-08-02$ & 7.1 & $14: 06: 37$ & $16.6^{\circ}$ & $-97.8^{\circ}$ \\
2 & $78-11-29$ & 7.8 & $19: 52: 50$ & $16.01^{\circ}$ & $-96.59^{\circ}$ \\
3 & $79-03-14$ & 7.0 & $11: 07: 15$ & $17.75^{\circ}$ & $-101.26^{\circ}$ \\
4 & $81-10-25$ & 7.3 & $03: 22: 16$ & $18.09^{\circ}$ & $-102^{\circ}$ \\
5 & $82-06-07$ & 6.9 & $06: 52: 33$ & $16.42^{\circ}$ & $-98.25^{\circ}$ \\
6 & $85-09-19$ & 8.1 & $13: 17: 49$ & $18.42^{\circ}$ & $-102.47^{\circ}$ \\
7 & $85-09-21$ & 7.6 & $01: 37: 14$ & $17.83^{\circ}$ & $-101.68^{\circ}$ \\
8 & $86-04-30$ & 7.0 & $07: 07: 19$ & $18.36^{\circ}$ & $-103.05^{\circ}$ \\
9 & $89-04-25$ & 6.0 & $14: 29: 03$ & $16.8^{\circ}$ & $-99.28^{\circ}$ \\
10 & $95-09-14$ & 7.3 & $14: 04: 33$ & $16.75^{\circ}$ & $-98.67^{\circ}$ \\
11 & $95-10-09$ & 7.5 & $15: 35: 54$ & $18.99^{\circ}$ & $-104.25^{\circ}$ \\
12 & $96-02-25$ & 6.9 & $03: 08: 19$ & $15.88^{\circ}$ & $-97.98^{\circ}$ \\
13 & $97-01-11$ & 6.9 & $20: 28: 26$ & $18.34^{\circ}$ & $-102.58^{\circ}$ \\
\hline
\end{tabular}


Las tablas A1-A6 incluidas en el Apéndice A de este artículo clasifican, de acuerdo a lo contemplado por el cuerpo principal de las NTCDS, los registros sísmicos empleados para el presente estudio; y resumen algunas de sus características más relevantes. Los movimientos del terreno utilizados para el estudio aquí reportado se establecieron por medio de rotar las componentes ortogonales registradas en cada estación hasta maximizar la intensidad de Arias del movimiento (Arias 1970). Los grupos de registros sísmicos se tomaron de un estudio realizado por Villa-Velázquez y Ruiz (2001), y se escalaron para ser congruentes con el nivel de aceleración máxima del terreno que establecen las NTCDS para las diferentes zonas.

En lo que sigue, se caracterizan las propiedades de los movimientos del terreno a través de una serie de espectros. Para obtener estos espectros, se consideraron sistemas de 1GL con periodos de vibración que van desde 0.1 hasta 5 segundos y que exhiben $5 \%$ de amortiguamiento crítico. En el caso de sistemas que exhiben comportamiento no lineal, se consideró un comportamiento elasto-plástico perfecto; y ductilidades últimas de 2,4 y 6 , que representan sistemas con capacidades de deformación limitada, moderada y alta, respectivamente.

Para cada una de las Zonas IIIa, IIIb, IIIc, y IIId, se procuró que los acelerogramas exhibieran un periodo dominante similar, de tal manera de no introducir variabilidad en los resultados en estos términos. En la tabla 2 se muestra el intervalo de periodos de terreno bajo consideración para cada zona. El periodo del terreno se definió como el periodo estructural que corresponde al valor pico dentro de un espectro de pseudo-aceleración. Para ejemplificar el efecto de promediar espectros obtenidos para sitios con periodo de terreno diferente, la figura 7 promedia los espectros elásticos de pseudo-aceleración para dos sitios con características dinámicas diferentes. Puede observarse que al promediar ambos espectros la forma espectral se suaviza, especialmente para la condición de resonancia, lo que puede resultar en una subestimación importante del efecto de amplificación dinámica. Bajo estas circunstancias, el espectro promedio no resulta representativo de ninguno de los sitios en estudio. Otra forma de evitar una cancelación de efectos al promediar espectros con distinto periodo dominante del terreno, consiste en normalizar el periodo estructural con respecto al periodo del terreno (Miranda y Ruiz-García 2002).

La figura 8, que muestra los espectros elásticos de pseudo-aceleración para los registros correspondientes a la Zona IIIb, ilustra que los movimientos del terreno han sido seleccionados para que no se presente el efecto de suavizado ilustrado en la figura 7. La figura 9, que ilustra la media de pseudoaceleración (línea continua) así como un intervalo de confianza establecido a partir de sumar y restar a la media una desviación estándar (líneas discontinuas), confirma a través de la baja dispersión en la forma espectral, que no se presenta un efecto de suavizado.

Tabla 2. Intervalo de periodos considerado en cada zona sísmica de terreno blando.

\begin{tabular}{ccc}
\hline Zona & Intervalo de Periodos (s) & Periodo promedio \\
\hline IIIa & $1.1-1.5$ & 1.3 \\
IIIb & $1.8-2.2$ & 2.0 \\
IIIc & $2.8-3.2$ & 3.0 \\
IIId & $3.6-4.4$ & 4.0 \\
\hline
\end{tabular}




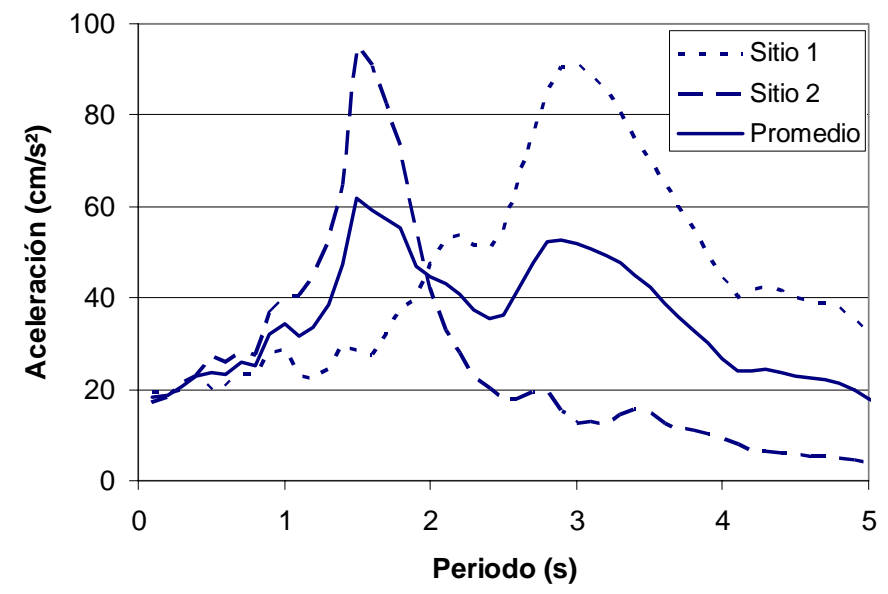

Figura 7. Suavizado de espectro elástico promedio de pseudo-aceleración para dos sitios con distinto periodo de terreno.

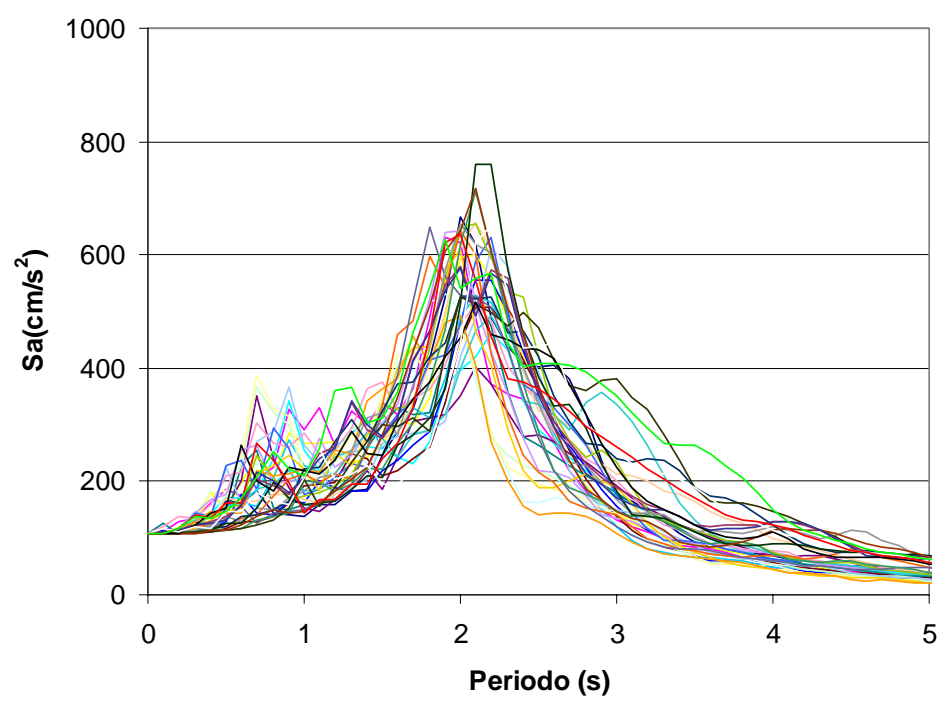

Figura 8. Espectros elásticos de pseudo-aceleración para movimientos correspondientes a la Zona IIIb.

\section{Resultados para la ductilidad objetivo}

La figura 10 muestra los valores de la ductilidad objetivo promedio obtenidos en cada zona a partir del índice de Park y Ang. Es importante recalcar que dichas ductilidades corresponden a la máxima ductilidad que los sistemas pueden desarrollar sin que el valor del índice de Park y Ang exceda un valor unitario. En estructuras desplantadas en terreno firme (Zona $I$ ), el valor medio de la ductilidad objetivo tiende a ser independiente del periodo estructural y exhibe dependencia con respecto a $\mu$. Cuando se toman en cuenta las demandas acumuladas de deformación plástica, se observan reducciones en la ductilidad objetivo respecto a la ductilidad última que pueden desarrollar las estructuras que van desde un $20 \%$ para $\mu$ de 2 , hasta un $35 \%$ para $\mu$ de 6 . Para el caso de las estructuras con periodos cortos, la 
Consideración explícita del daño acumulado en el diseño sísmico de estructuras a través de factores de reducción ...

influencia de la acumulación del daño por demandas plásticas es casi nula (esta última observación aplica para las seis zonas bajo consideración).

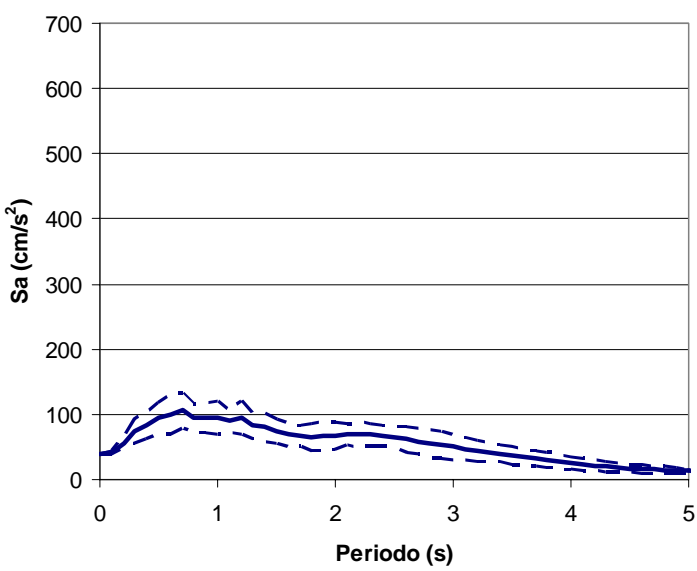

a) Zona $I$

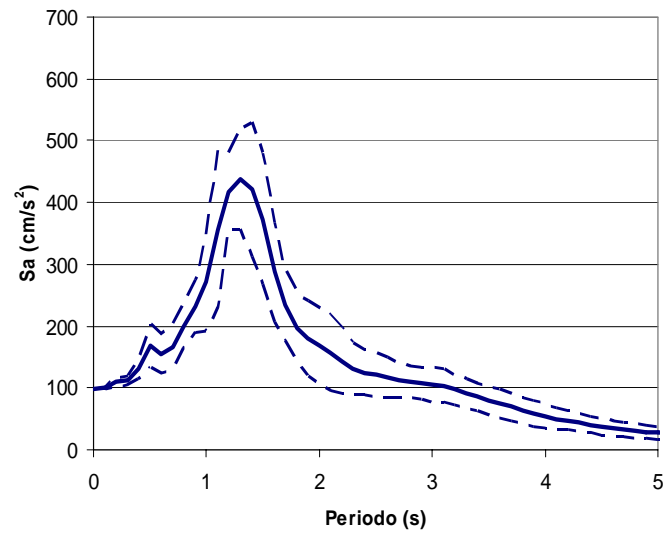

c) Zona IIIa

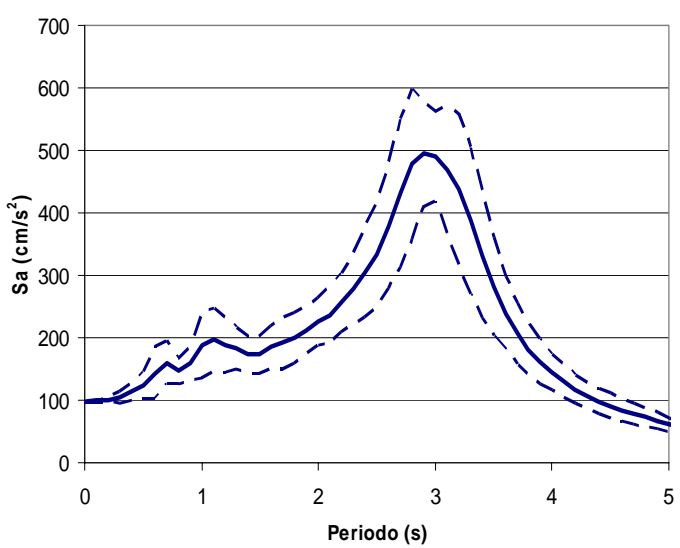

e) Zona IIIc

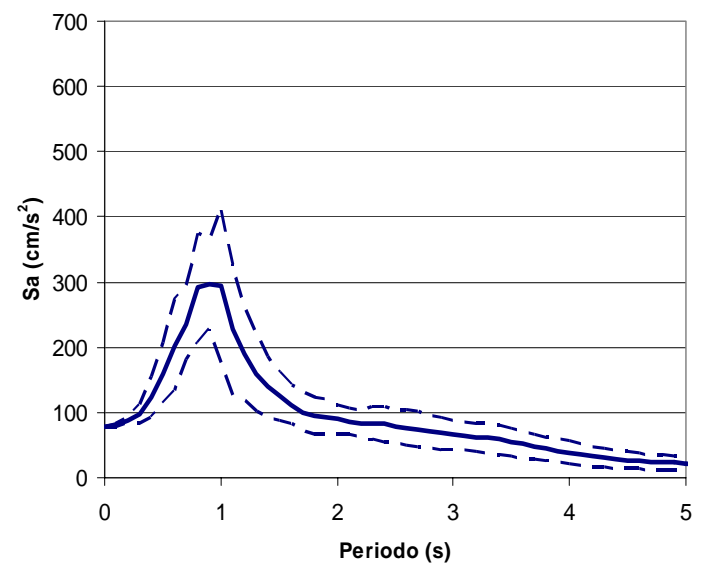

b) Zona $I I$

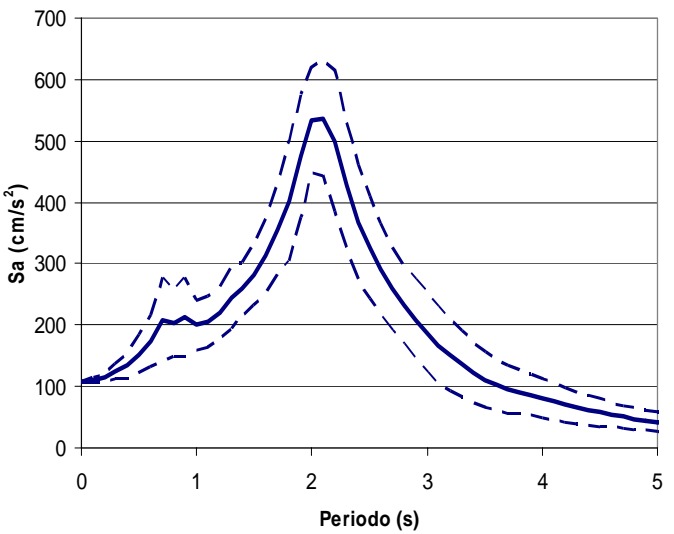

d) Zona IIIb

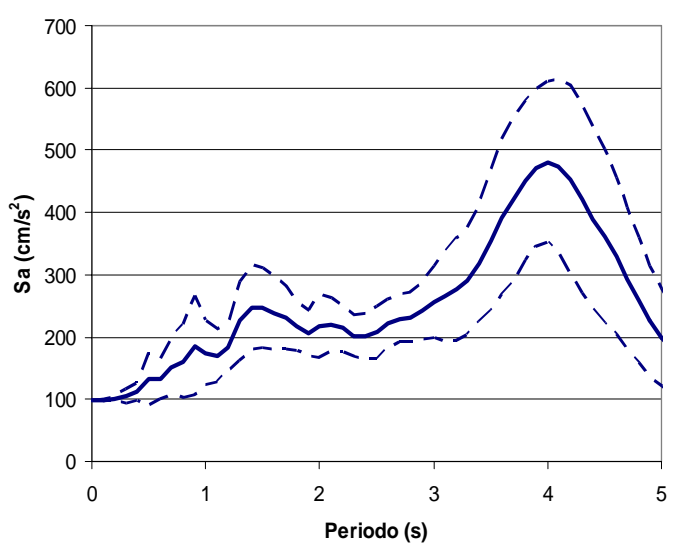

f) Zona IIId

Figura 9. Espectros elásticos de pseudo-aceleración correspondientes a las diferentes zonas sísmicas del Valle de México. 


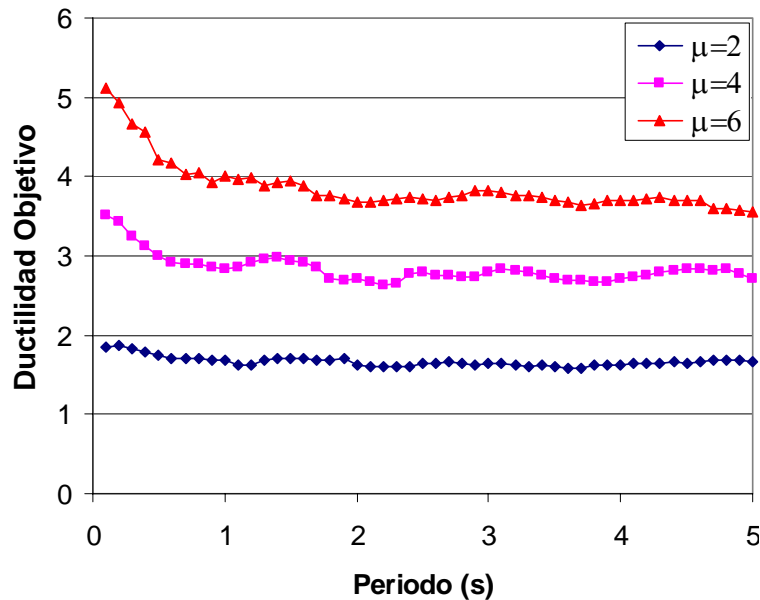

a) Zona $I$

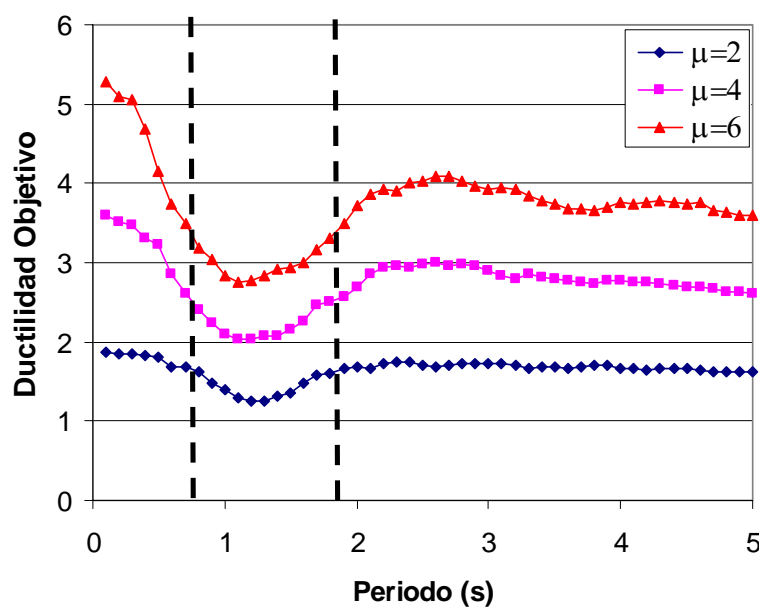

c) Zona IIIa

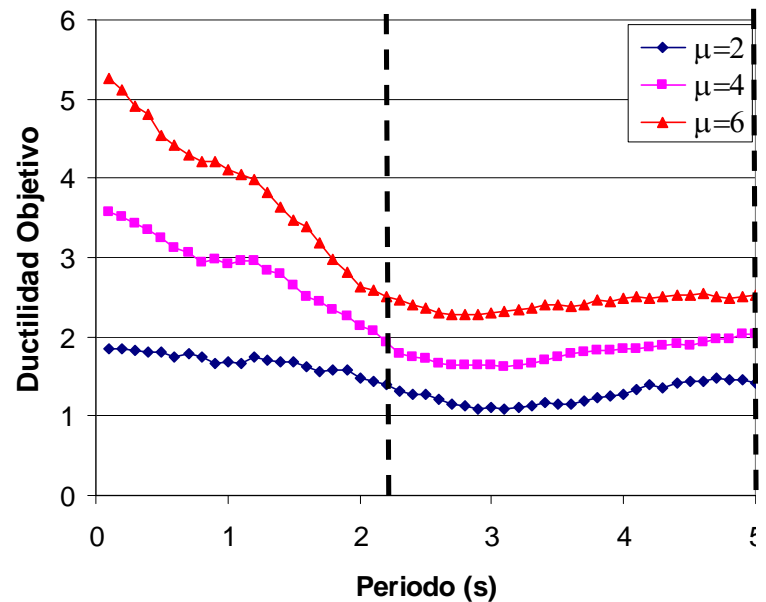

e) Zona IIIC

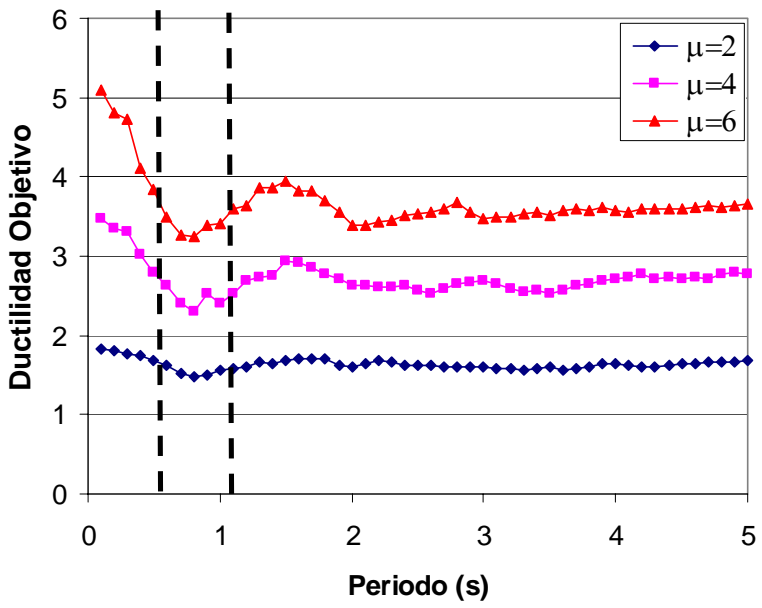

b) Zona $I I$

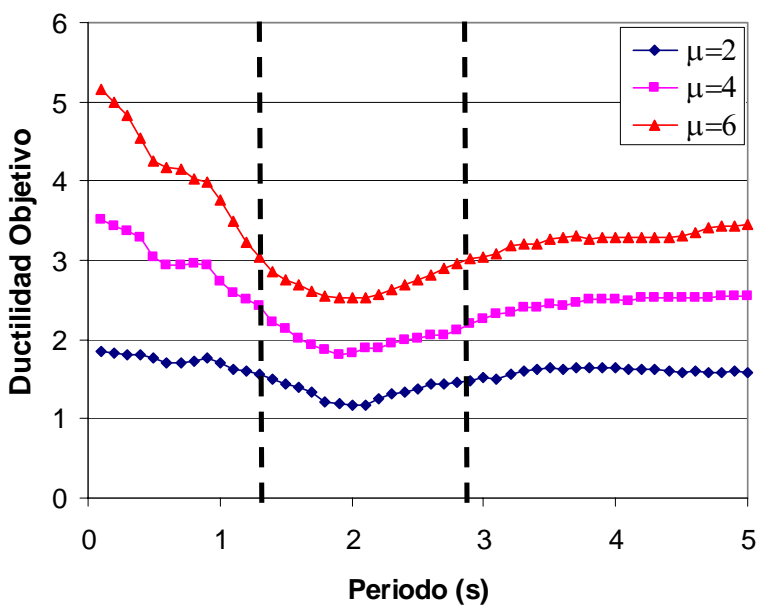

d) Zona $I I I b$

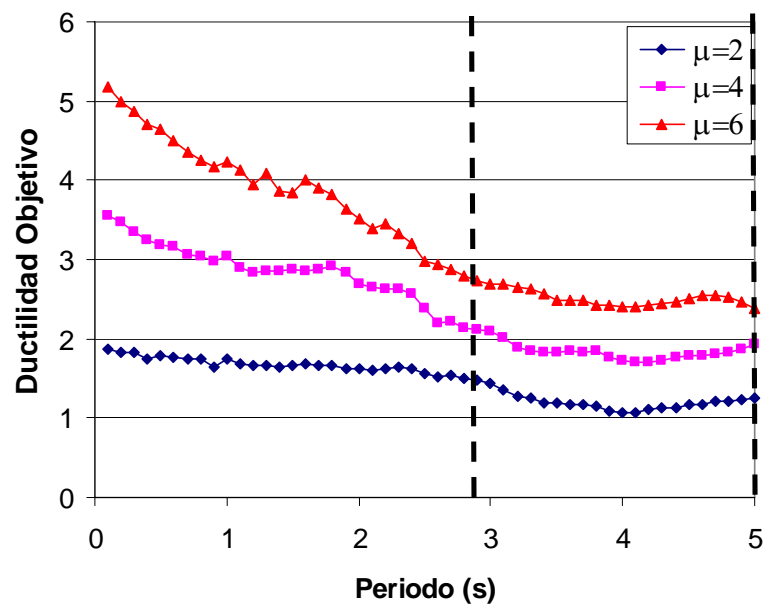

f) Zona IIId

Figura 10. Ductilidades objetivo correspondientes a las diferentes zonas sísmicas del Valle de México. 
En las zonas que corresponden a terrenos de transición y del lago se observa una influencia importante de la relación entre el periodo estructural y el periodo del terreno en el valor de la ductilidad objetivo. Las estructuras con periodos cercanos al periodo dominante del terreno sufren las mayores reducciones en términos de ductilidad (área entre líneas discontinuas de cada figura), y se llegan a obtener valores de ductilidad objetivo del orden del $50 \%$ al $60 \%$ de $\mu$. Estos porcentajes son del orden de los sugeridos por Bertero (1997) y Panagiotakis y Fardis (2001) para el caso de estructuras o elementos sujetos a altas demandas de energía. Dentro de este contexto, es posible observar en casos extremos valores de ductilidad objetivo menores que el $50 \%$ de $\mu$. Dado que en estructuras cuyo periodo se aleja del periodo dominante del terreno, considerar reducciones de la ductilidad del orden de las identificadas en la condición de resonancia suele resultar en diseños demasiado conservadores, sería importante seguir utilizando para su diseño el valor de $\mu$ como medida de la ductilidad máxima que puede desarrollar el sistema.

Las figuras $10 \mathrm{~b}$ a $10 \mathrm{f}$ indican que en algunos casos se presentan disminuciones importantes de ductilidad aún para estructuras con periodos alejados del periodo del terreno (particularmente en estructuras desplantadas en suelos muy blandos y con periodos de vibración largos). Note que a medida que el periodo dominante del terreno se incrementa, las deformaciones plásticas acumuladas tienden a exhibir una influencia significativa en un mayor intervalo de periodos estructurales (observe el incremento del intervalo de periodos abarcados por la líneas discontinuas en cada figura, sobre todo para las Zonas IIIc y IIId).

Varios investigadores sugieren que tendencias como las ilustradas en la figura 10, pueden entenderse mejor si en los espectros que se establecen para suelos blandos, el eje de las abscisas considera el periodo estructural normalizado por el periodo dominante del terreno (Miranda 1993, Miranda y RuizGarcía 2002, Avilés y Pérez Rocha 2007). La figura 11 muestra para periodo normalizado algunos de los resultados presentados en la figura 10 para $\mu$ de 4 . La figura 11 hace evidente la fuerte dependencia de la ductilidad objetivo con respecto al periodo del suelo; es decir, las mayores reducciones en cuanto a capacidad de deformación ocurren para estructuras con periodos cercanos al periodo del terreno. Se confirma además que las reducciones en la ductilidad objetivo son menos significativas a medida que el periodo estructural se aleja del periodo del terreno, y se observa, una vez más, que el alto contenido de energía de los movimientos del terreno en la Zona del Lago afecta un mayor intervalo de periodos conforme se incrementa el periodo dominante de dichos movimientos. Respecto a esto último, note en la figura 11 que mientras las curvas de ductilidad objetivo son muy similares entre sí para periodos normalizados menores que uno, las ductilidades tienden a exhibir valores significativamente diferentes para periodos normalizados mayores que uno. En particular, note que las ductilidades para valores de periodo normalizado mayor que 1.7 van desde tres para la Zona IIIa, hasta dos para la Zona IIIc. Puede decirse que en un intervalo de periodos estructurales que va de $0.75 T_{s}$ hasta $1.25 T_{s}$ (donde $T_{s}$ es el periodo dominante del terreno) la ductilidad objetivo se reduce aproximadamente de $50 \%$ a $60 \%$ de la ductilidad última, y que conforme se incrementa el periodo dominante del terreno, el límite superior indicado tiende incrementarse de manera importante.

La figura 12 muestra el coeficiente de variación para la ductilidad objetivo correspondiente a cada zona y una ductilidad última de 4. En general se observa poca dispersión en la ductilidad objetivo, caracterizada por coeficientes de variación que caen en un intervalo que va desde valores cercanos a cero hasta valores de 0.2. Note que el nivel de dispersión es bastante similar para todas las zonas bajo estudio. 


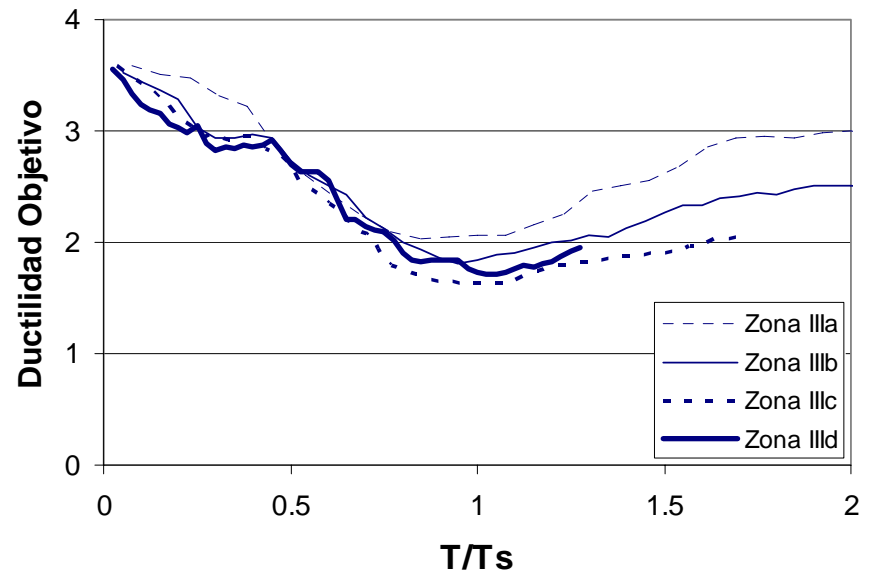

Figura 11. Ductilidades objetivo correspondientes a las zonas de suelo blando del valle de México, $\mu=4$.

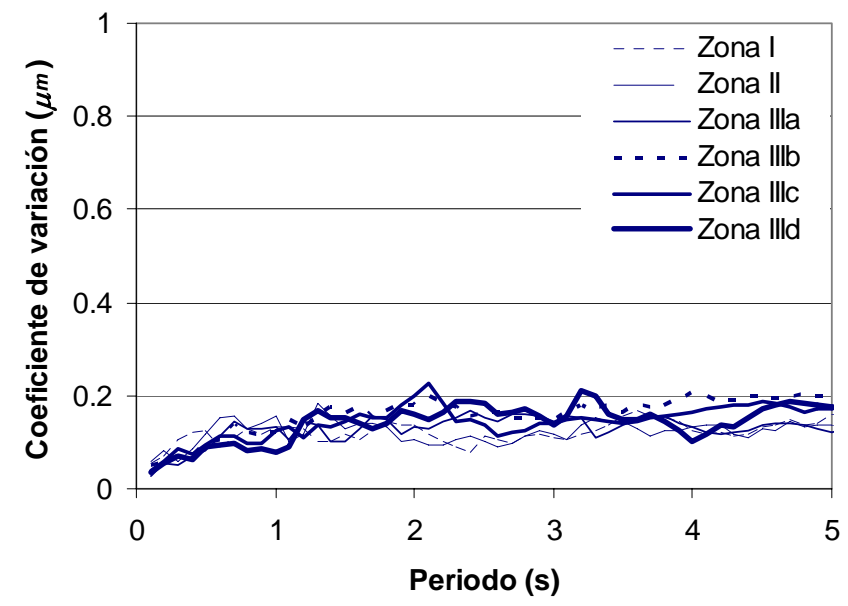

Figura 12. Coeficiente de variación de la ductilidad objetivo para cada zona bajo consideración, $\mu=4$.

\section{FACTORES DE REDUCCIÓN DE RESISTENCIA QUE INCORPORAN LA INFLUENCIA DEL DAÑO ACUMULADO}

Para considerar el comportamiento plástico de las estructuras, los códigos de diseño sísmico utilizan factores de reducción de resistencia por ductilidad. Dada su importancia para el diseño sismo-resistente, se han invertido una gran cantidad de estudios en esta dirección (Miranda 1993, Miranda y Bertero 1994, Ordaz y Pérez-Rocha 1998, Lam et al. 1998, Lee, Sanh y Ho 1999). El factor de reducción por ductilidad $\left(R_{\mu}\right)$ se define como la relación entre la mínima resistencia requerida para mantener la estructura elástica, $F_{y}(\mu=1)$, y la resistencia requerida para que la misma desarrolle una ductilidad $\mu_{i}, F_{y}\left(\mu=\mu_{i}\right)$ (ver ecuación 9 y figura 13). 


$$
R_{\mu}=\frac{F_{y}(\mu=1)}{F_{y}\left(\mu=\mu_{i}\right)}
$$

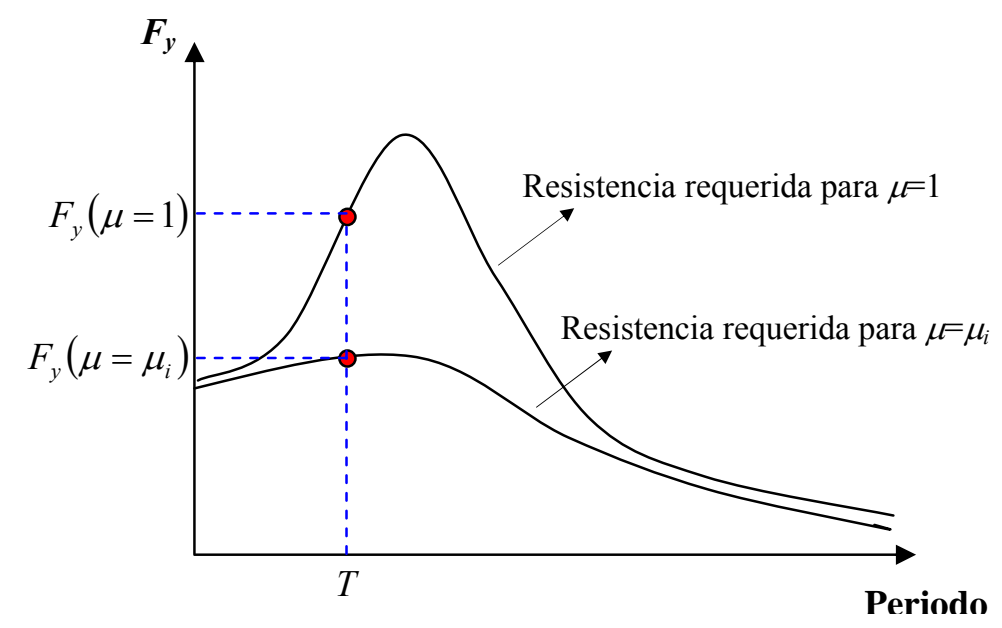

Figura 13. Obtención del factor de reducción por ductilidad.

Aún cuando el factor $R_{\mu}$ ha sido bien estudiado, falta incorporar en él información acerca de la capacidad resistente extra que deben poseer las estructuras sismo-resistentes para acomodar demandas severas de deformación plástica. Para atender explícitamente esta capacidad extra, en este trabajo se obtienen factores de reducción de resistencia asociados a las ductilidades objetivo obtenidas a partir del índice de Park y Ang:

$$
R_{\mu A}=\frac{F_{y}(\mu=1)}{F_{y}\left(\mu=\mu_{m i}\right)}
$$

donde $R_{\mu A}$ es el factor de reducción por ductilidad que incorpora información acerca de la influencia de las demandas acumuladas por comportamiento plástico, y $F_{y}\left(\mu=\mu_{m_{i}}\right)$ la resistencia requerida para que se desarrolle la ductilidad objetivo $\mu_{m i}$ de acuerdo al índice de daño de Park y Ang. El cociente entre $R_{\mu}$ y $R_{\mu A}$ representa el factor de incremento en la resistencia lateral que debe considerarse para diseñar la estructura bajo consideración explicita del daño acumulado:

$$
F_{I A}=\frac{R_{\mu}}{R_{\mu A}}
$$

En la siguiente parte de este trabajo, se obtiene el factor de incremento de resistencia lateral para considerar el efecto de las demandas acumuladas de deformación plástica en las distintas zonas del valle de México. 
Valores de $F_{I A}$ para el Valle de México

Los factores de incremento de la resistencia lateral $F_{I A}$ para las distintas zonas, ductilidades y periodos bajo consideración se ilustran en la figura 14. Conforme a lo que se esperaba, para las Zonas $I$ y $I I$, que corresponden a terreno firme y de transición, $F_{I A}$ se mantiene constante en un largo intervalo de periodos. En el caso de terrenos en la Zona del Lago, es notoria la influencia del periodo dominante del terreno en los incrementos de resistencia lateral de las estructuras. De manera similar al caso de $\mu_{m}$, las demandas acumuladas de deformación plástica exhiben una influencia importante en un intervalo mayor de periodos conforme se incrementa el periodo dominante del terreno. En las Zonas $I$ y $I I$, el incremento de resistencia lateral oscila entre 20 y $60 \%$, y su valor depende de la ductilidad última de la estructura. En particular, un incremento en esta ductilidad se ve reflejado en un mayor incremento relativo de la resistencia lateral para tomar en cuenta el efecto del daño acumulado. Se observa como en las zonas de terreno blando (IIIa y IIIb) puede llegar a ser necesario incrementar la resistencia lateral al doble o más de la requerida según un criterio que no contemple el efecto de daño acumulado para estructuras con periodos similares al periodo del terreno. Estos resultados son congruentes con los reportados para $\mu_{m}$ en la sección anterior, ya que la reducción en la ductilidad permisible en estructuras ubicadas en suelos blandos y con periodos cercanos al periodo dominante del terreno era del orden del $50 \%$ o mayor.

Normalmente los espectros de diseño que se plantean en los códigos tienden a ser conservadores. El conservadurismo implícito en los estudios analíticos que se llevan a cabo para sustentar dichos espectros suele complementarse con el uso de juicio ingenieril para dar lugar a diseños de resistencia conservadores. Dentro de un contexto en que es difícil establecer el nivel de conservadurismo asociado al uso de las NTCDS, los valores de $F_{I A}$ mostrados en la figura 14 para las Zonas $I$ y II no aportan elementos suficientes para plantear un incremento en las ordenadas espectrales de pseudo-aceleración. En el caso de la Zona $I I I, F_{I A}$ alcanza valores cercanos a tres (figura 14d). Dado que es difícil pensar que una disposición reglamentaria, por más conservadora que sea, plantee una resistencia de diseño que sea tres veces la requerida, es posible decir que para estructuras ubicadas en la Zona del Lago y cuyo periodo estructural esté cercano al periodo dominante del terreno, es necesario replantear, dentro del contexto de las NTCDS, la manera en que se estima su resistencia lateral de diseño.

\section{Comparación de factores de reducción que consideran daño acumulado y los propuestos por el RCDF-2004}

Resulta de interés comparar los factores de reducción de resistencia obtenidos con y sin consideración explícita del daño acumulado con aquellos incluidos en el Apéndice A de la última versión de las NTCDS. Vale la pena hacer notar que los factores de reducción incluidos en el Apéndice A se establecieron a partir de un criterio que considera el desplazamiento máximo como único indicador de daño en sistemas con comportamiento elasto-plástico perfecto (Ordaz et al. 2003). Debido a que el Apéndice A no contempla una subdivisión del tipo Zona I, II, IIIa, etc., la comparación de los factores de reducción obtenidos aquí con aquellos planteados por dicho apéndice debe hacerse para sitios específicos. Para ilustrar la comparación de factores, en este artículo se considera un sitio con periodo dominante de dos segundos. La figura 15 compara, para una ductilidad última de 4 , los factores obtenidos mediante el Apéndice A para $T_{s}$ de 2 segundos, y aquellos obtenidos para la Zona $I I I b$ cuando $T_{s}=2 \mathrm{~s}$ y no se consideran los efectos acumulados de deformación plástica. Puede observarse la similitud que exhiben ambos juegos de factores de reducción para un amplio intervalo de periodos estructurales. Es posible decir a partir de la comparación, que bajo la consideración de la demanda máxima de deformación plástica, el Apéndice A presenta una definición razonable de los factores de reducción por resistencia (la aparente 
sobrestimación para periodos cortos y largos puede explicarse a partir de la forma especificada para los espectros elásticos de resistencia de diseño, los cuales exhiben valores conservadores de resistencia en dichas zonas de periodo).

La figura 16 muestra que una vez que se considera el efecto del daño acumulado en la determinación de los factores de resistencia, las reducciones al espectro de diseño elástico deben ser mucho menores que las consideradas por la normatividad actual. En congruencia con lo indicado en secciones previas, se observan diferencias hasta de 50\% para estructuras ubicadas en la Zona III y cuyo periodo se encuentra cercano al periodo dominante del terreno. Los resultados que se muestran aportan información que debe complementar al enfoque actual para permitir una toma de decisiones adecuada en cuanto al diseño sísmico de estructuras desplantadas en la Zona del Lago del Distrito Federal.

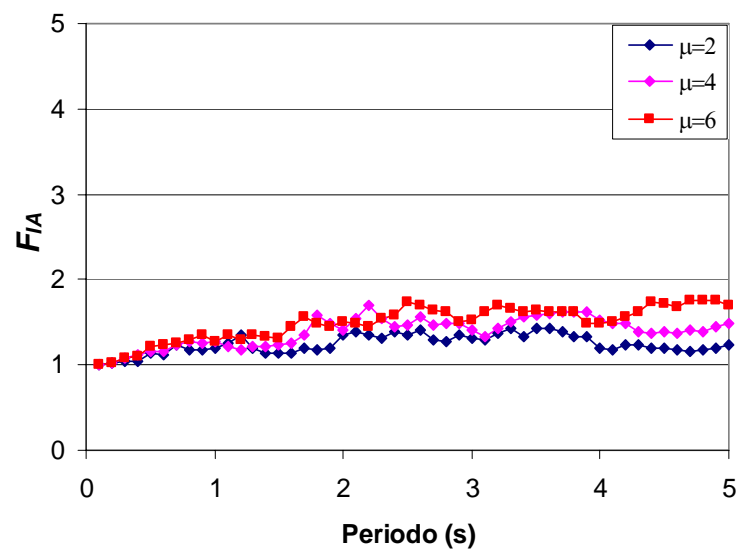

a) Zona $I$

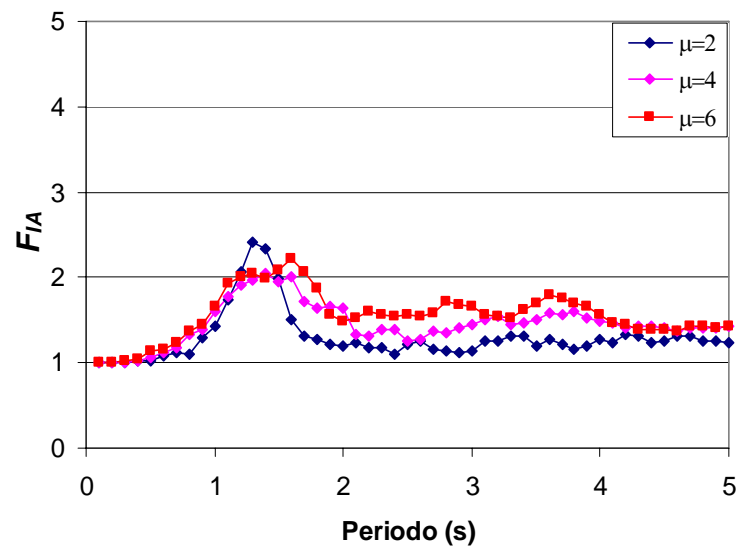

c) Zona IIIa

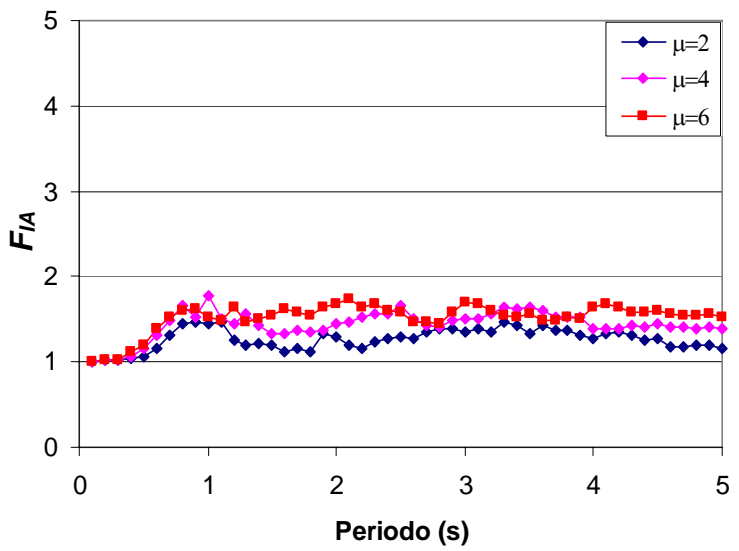

b) Zona $I I$

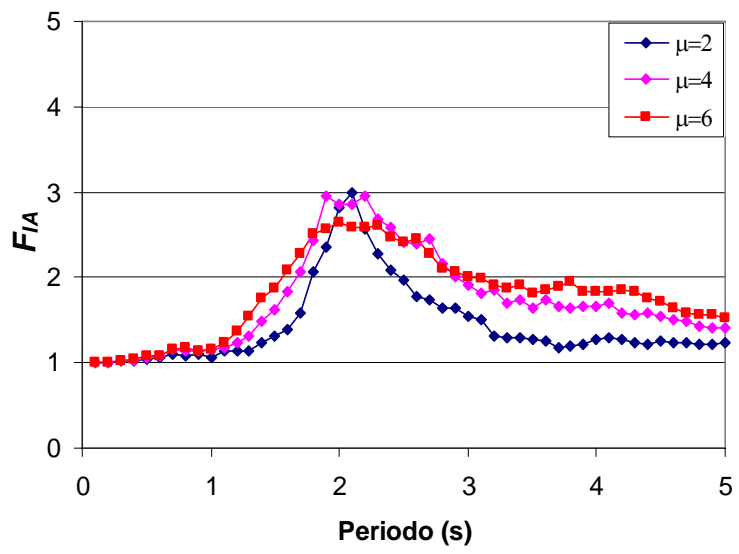

d) Zona $I I I b$

Figura 14. Valores medios de $F_{I A}$ correspondientes a diferentes zonas sísmicas del Valle de México. 


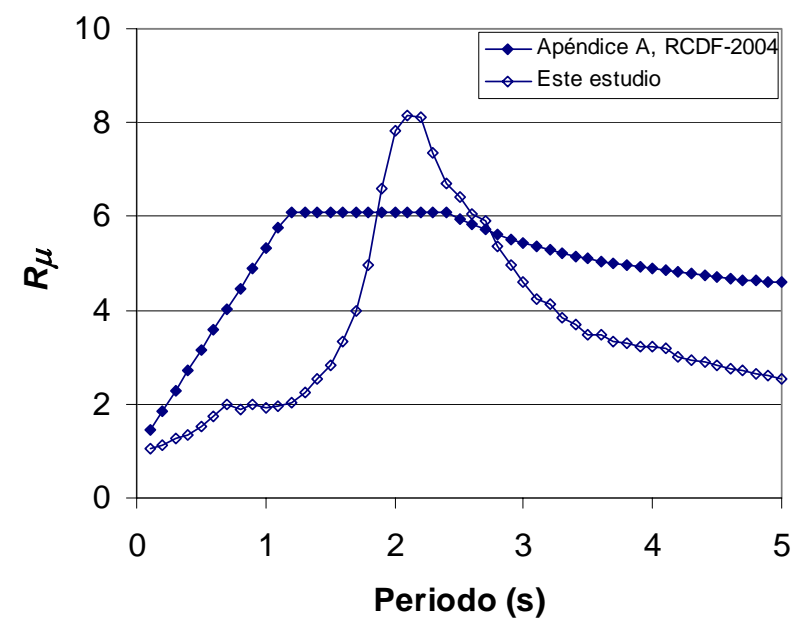

Figura 15. Factores de reducción por ductilidad, Apéndice A versus este estudio sin consideración de daño acumulado, $\mu=4$ y $T_{s}=2$ segundos.

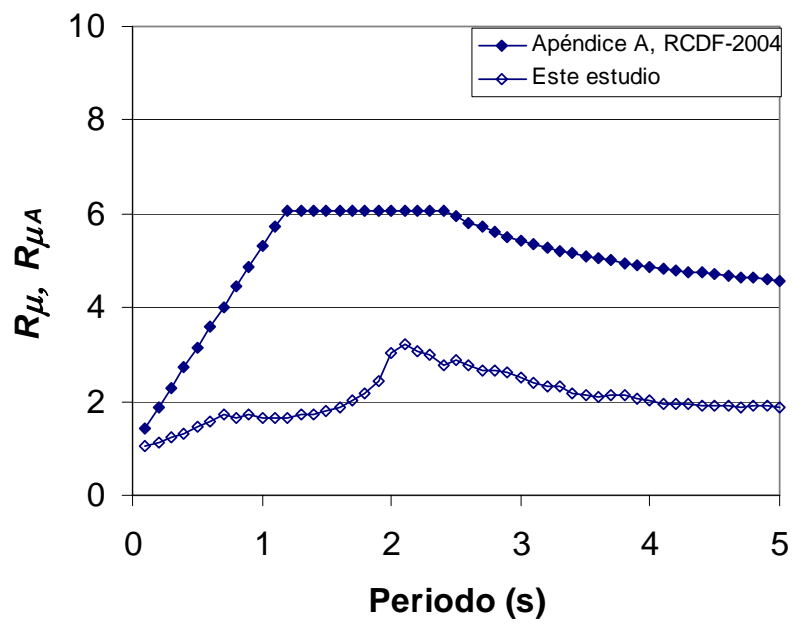

Figura 16. Factores de reducción por ductilidad, Apéndice A versus este estudio con consideración de daño acumulado, $\mu=4$ y $T_{s}=2$ segundos.

\section{ESTRUCTURAS CON DEGRADACIÓN DE RIGIDEZ Y RESISTENCIA}

Aunque las comparaciones establecidas a través de las figuras 15 y 16 son congruentes en términos del comportamiento elasto-plástico perfecto considerado por el planteamiento hecho por el Apéndice A y por el alcance de estudio que se presenta en este artículo, es necesario considerar que varias de las estructuras sismo-resistentes que se diseñan y construyen exhiben comportamiento histerético degradante. En particular, el comportamiento cíclico de las estructuras de concreto reforzado y mampostería es muy diferente al elasto-plástico, y entonces es importante discutir las implicaciones de utilizar modelos bilineales para establecer la resistencia lateral de diseño de estructuras sismo-resistentes degradantes. 
Varios estudios sugieren que para excitaciones sísmicas generadas en suelos blandos, las demandas sísmicas en sistemas que exhiben degradación de rigidez pueden llegar a ser significativamente diferentes que aquellas estimadas de espectros establecidos a partir de comportamiento elasto-plástico perfecto (Terán 1996, Miranda y Ruiz 1999, Espinoza y Terán 2000, Miranda y Ruiz 2002, Terán y Espinoza 2003, Ruiz y Miranda 2004). En particular, mientras que Miranda y Ruiz-García (2002) observan que para estructuras degradantes ubicadas en suelos blandos y con periodo estructural menor que el periodo del terreno, la resistencia lateral requerida puede llegar a ser hasta $20 \%$ mayor de aquella obtenida mediante un sistema no-degradante, Terán-Gilmore y Espinoza (2008) encontraron para la Zona del Lago que ese porcentaje puede alcanzar hasta el $30 \%$. En caso de sistemas degradantes con periodo estructural mayor que el periodo del terreno, los requerimientos de resistencia lateral son menores que las de sus contrapartes elasto-plásticos. Lo anterior implica que la resistencia lateral de diseño leída de un espectro elasto-plástico debe modificarse antes de ser aplicada a una estructura con comportamiento degradante. Existen propuestas para corregir la ordenada espectral leída de un espectro elasto-plástico de pseudoaceleración para el caso de estructuras degradantes que no exhiben adelgazamiento de su ciclo histerético, por ejemplo (Terán-Gilmore y Espinoza 2008):

$$
F_{D E G}(T)=\frac{S_{a D E G}}{S_{a E P P}}=\frac{1}{2+5\left|\frac{T}{0.5 T_{S}}-1\right|^{5}}+0.8
$$

donde $F_{D E G}$ es el factor de corrección por comportamiento degradante, y $S_{a D E G}$ y $S_{a E P P}$ las ordenadas espectrales de pseudo-aceleración correspondientes a comportamiento degradante y elasto-plástico perfecto, respectivamente. En caso de que la estructura exhiba comportamiento elasto-plástico, $F_{D E G}$ debe ser igual a uno.

\section{OBSERVACIONES Y RECOMENDACIONES PARA CONSIDERAR LOS EFECTOS DEL DAÑO ACUMULADO}

De acuerdo con lo planteado hasta el momento, la resistencia de diseño de una estructura sismorresistente debe establecerse conforme a lo siguiente:

$$
S_{a d}=\frac{S_{a}(\mu=1)}{R_{\mu} R} F_{I A} F_{D E G}
$$

donde $S_{a d}$ es la pseudo-aceleración de diseño, $S_{a}(\mu=1)$ la ordenada espectral elástica, $R_{\mu}$ el factor de reducción debido a comportamiento plástico (ecuación 9), $F_{I A}$ el factor que considera las demandas acumuladas de deformación plástica (ecuación 11), $R$ el factor de reducción por sobre-resistencia, y $F_{D E G}$ el factor que considera el comportamiento histerético de la estructura (ecuación 12). De los factores involucrados en la ecuación 13, el Apéndice A considera explícitamente $R_{\mu}$ y $R$. En cuanto a los restantes, se ha hecho una propuesta para que la nueva versión del Manual de Obras Civiles de la Comisión Federal de Electricidad considere explícitamente $F_{D E G}$ en la estimación de la resistencia lateral de las estructuras sismo-resistentes. En cuanto al factor $F_{I A}$, los resultados presentados en este artículo muestran la importancia de considerar las demandas acumuladas de comportamiento plástico en el diseño sísmico de las estructuras desplantadas en el Valle de México (especialmente para aquellas ubicadas en la Zona del Lago y cuyos periodos se encuentran cercanos al periodo dominante del terreno). 


\section{La estimación de las demandas de energía plástica}

En cuanto al modelo utilizado para estimar las demandas de energía plástica puede decirse lo siguiente:

- Los sistemas considerados en este trabajo se han supuesto sobre base rígida. Al respecto, surge la duda de si el cambio en las propiedades dinámicas y el amortiguamiento adicional que exhibe un sistema modelado sobre base flexible alteran de manera importante sus demandas sísmicas, particularmente las de deformación plástica acumulada. Dadas las características del suelo en el Valle de México, varios investigadores han estudiado el efecto de la interacción suelo-estructura en las demandas sísmicas que exhiben sistemas simples desplantados en sus diferentes zonas (Tarquis y Roesset 1988, Bazán et al. 1992, Pérez-Rocha y Avilés 2003, 2007). De especial interés para esta discusión es el trabajo desarrollado por Rodríguez y Montes (1998), ya que consideró el efecto de dicha interacción en las demandas de energía plástica. Rodríguez y Montes concluyen que: “... en un intervalo amplio de periodos de edificios de varios niveles sobre base flexible, representativos de los desplantados sobre la antigua zona del lago de la ciudad de México, las demandas de desplazamientos relativos y de energía histerética en este tipo de sistemas se pueden evaluar empleando resultados del caso sobre base rígida, para lo cual es necesario emplear el periodo amplificado correspondiente del caso sobre base flexible". En un trabajo reciente, Avilés y Pérez-Rocha (2007) observan que las demandas de energía para el caso de base rígida se incrementan o reducen por los efectos de interacción en función de la relación que guardan los periodos estructural y del terreno.

- Las estructuras reales tienden a exhibir sobre-resistencia en términos de sismo-resistencia. Esta capacidad extra se debe a muchas razones entre las cuales pueden mencionarse las siguientes (Miranda y Bertero 1989, Uang 1991, Paulay 1996):

a) El planteamiento de los espectros de resistencia de diseño suele contemplar que las estructuras son capaces de desarrollar una resistencia lateral mayor que la considerada durante su diseño. Al respecto, el valor de sobre-resistencia considerado para fines de diseño tiende a ser conservador.

b) Existen varios elementos, tanto estructurales como no estructurales, cuya contribución a la sismo-resistencia suele ignorarse con fines de diseño.

c) La diferencia entre la respuesta de las estructuras cuando se les sujeta a cargas de naturaleza estática y dinámica. Por lo general, los modelos estructurales se plantean a partir de propiedades establecidas de estudios que consideran cargas de naturaleza pseudo-estática. Las propiedades estructurales en términos de rigidez y resistencia de las estructuras sismo-resistentes pueden incrementarse de manera importante para el caso dinámico con respecto a sus valores estáticos.

Lo anterior implica que las estructuras reales tienden a exhibir una mayor resistencia que la contemplada durante su diseño (Terán 1998; Terán y Espinoza, 2008), y por tanto, que sus demandas de deformación plástica máxima y acumulada tenderán a ser menores que las estimadas analíticamente.

- Las estructuras reales exhiben una importante variabilidad en cuanto a sus propiedades dinámicas, tanto en términos de periodos estructurales como de amortiguamiento. Por ejemplo, el periodo 
fundamental de vibración de una estructura real evoluciona, más allá de lo que pueden predecir las herramientas analíticas con las que se cuenta hoy en día, no solo de excitación sísmica a excitación sísmica, sino incluso durante el transcurso de una excitación sísmica (Anderson et al. 1991, Muria et al. 1995, 2000). En cuanto al amortiguamiento, es común asociar a los espectros de diseño amortiguamientos de $5 \%$ del crítico, cuando la evidencia que se tiene indica que los materiales estructurales de mayor uso en México exhiben niveles de amortiguamiento que exceden de manera importante dicho valor durante excitaciones sísmicas severas (Chopra 2001). Mientras que en términos del periodo, lo anterior implica cierta dificultad para predecir adecuadamente las demandas sísmicas en estructuras reales, en términos de amortiguamiento las demandas de energía plástica esperadas en una estructura real debieran ser ligeramente menores a las estimadas analíticamente.

En cuanto a los movimientos del terreno utilizados para obtener los resultados analíticos mostrados aquí, puede decirse lo siguiente:

- El único registro utilizado aquí que verdaderamente representa el contenido de energía de un movimiento del terreno generado en la Zona del Lago del Valle de México durante un evento sísmico extremo es el acelerograma SCT EO registrado durante 1985. Con excepción de éste, los demás acelerogramas utilizados en el estudio se escalaron para que representaran movimientos del terreno generados en eventos sísmicos extremos. Al respecto, se observa que cuando se escalan linealmente acelerogramas poco intensos, estos presentan un contenido de energía que es mayor que el exhibido por el registro SCT EO 1985. Lo mismo puede decirse de los acelerogramas sintéticos generados a partir de algoritmos que generan movimientos del terreno de gran intensidad en la Zona del Lago del D.F. a partir de semillas que corresponden a movimientos de baja intensidad registrados en dicha zona (Quiroz y Terán 2008). Por el momento, es difícil definir si el contenido de energía de los acelerogramas escalados linealmente o de acelerogramas sintéticos generados a partir de semillas de baja intensidad es representativo de lo que se espera durante un evento sísmico extremo.

A partir de la discusión desarrollada en esta sección, es posible afirmar que un estudio analítico tenderá a sobreestimar las demandas acumuladas de deformación plástica en las estructuras reales, y que existe una alta incertidumbre en cuanto a la estimación de dichas demandas debido a la dificultad de modelar adecuadamente varios de los elementos que contribuyen, de manera explícita o implícita, a la sismo-resistencia. Sin embargo, es necesario mencionar que los estudios llevados a cabo por varios investigadores mexicanos indican una alta probabilidad de que varias de las estructuras desplantadas en el D.F., particularmente las ubicadas en la Zona del Lago, exhiban durante sismos severos altas demandas de deformación plástica acumulada que no están siendo consideradas durante su diseño sismo-resistente (Rodríguez y Ariztizabal 1999, Huerta y Reinoso 2002, Bojórquez y Ruiz 2004, Arroyo y Ordaz 2007).

\section{Recomendaciones para incorporar el daño acumulado al diseño sismo-resistente de las estructuras}

Algunas secciones previas a esta han discutido la tendencia a que los modelos analíticos sobrestimen las demandas de energía y a que los indicadores de daño sobrestimen los efectos de las demandas acumuladas de deformación plástica en las estructuras. Contextualizando los resultados obtenidos en este trabajo se sugiere lo siguiente:

- Dada la poca influencia que presenta el daño acumulado en las estructuras desplantadas en las zonas de suelo firme y de transición (Zonas I y II), se sugiere, conforme a lo indicado en la tabla 3 , utilizar ductilidades objetivo que sean del orden de 80 a $90 \%$ de la ductilidad última. Esto 
implica incrementar las demandas de resistencia a partir de las cuales se establecen los espectros de diseño de un 10 a $20 \%$.

- Conforme a lo indicado en la tabla 3, especial atención requiere el diseño sismo-resistente de las estructuras ubicadas en suelos blandos. En caso de que el periodo de la estructura se aleje del periodo dominante del terreno, la reducción de ductilidad debe ser del orden de 10 a $30 \%$. Por el contrario, en caso en que el periodo de la estructura se acerque al valor de $T_{S}$, la ductilidad objetivo debiera reducirse hasta en $40 \%$ con respecto a la ductilidad última. Para esto puede considerarse, como lo sugiere la tabla 3 , un intervalo de periodos que va desde $0.5 T_{s}$ hasta $1.5 T_{s}$.

Dado que las recomendaciones anteriores dan lugar a niveles de diseño muy costosos para las estructuras ubicadas en las diferentes sub-zonas de la Zona del Lago y cuyo periodo esté cercano al periodo dominante del terreno, es recomendable que para este tipo de estructuras se utilicen dispositivos especiales, tal como los disipadores de energía, que permitan una disipación de energía más controlada que impida el daño acumulado excesivo en los elementos estructurales.

Tabla 3. Reducciones de ductilidad recomendadas para incorporar el efecto de las demandas acumuladas de deformación plástica.

\begin{tabular}{ccc}
\hline Zona & \multicolumn{2}{c}{$\%$ reducción de $\mu$} \\
\cline { 2 - 3 } & $T$ lejano a $T_{s}$ & $0.5 T_{s} \leq T \leq 1.5 T_{s}$ \\
\hline$I$ & $10-20$ & $10-20$ \\
$I I$ & $10-20$ & $10-20$ \\
III & $10-20$ & $20-30$ \\
IIIb & $20-30$ & $30-40$ \\
IIIc & $20-30$ & $30-40$ \\
IIId & $20-30$ & $30-40$ \\
\hline
\end{tabular}

\section{CONCLUSIONES}

Se evaluó a partir del índice de daño de Park y Ang la ductilidad objetivo para varios sistemas simples que representan estructuras ubicadas en las distintas zonas del Valle de México. Los resultados obtenidos indican que puede llegar a presentarse influencia importante del daño acumulado en el desempeño sísmico de dichos sistemas. Para estructuras desplantadas en terreno firme se observó, independientemente de su periodo, un efecto de bajo a moderado del daño acumulado. Bajo estas circunstancias se recomienda un incremento que oscila del 10 al 20\% en las demandas de resistencia lateral que han sido consideradas para plantear los espectros de diseño de resistencia.

En el caso de la Zona del Lago, se observó una influencia significativa de las demandas acumuladas de deformación plástica en estructuras con periodos cercanos al periodo dominante del terreno. Además, se observó que conforme se incrementa el valor del periodo dominante del terreno, las demandas de energía tienden a afectar estructuras que se encuentran en un mayor intervalo de periodos. Dada la severidad del daño acumulado en esta zona, se sugiere hacer reducciones de un 30 a $40 \%$ en los niveles de ductilidad a partir de los cuales se obtienen las resistencias de diseño.

Por un lado, es importante mencionar que los resultados presentados aquí son fuertemente dependientes de la capacidad del índice de daño de Park y Ang para evaluar el daño estructural en sistemas sismo-resistentes. Por otro lado, es importante mencionar que las resistencias de diseño derivadas 
del uso de dicho índice son muy similares a aquellas derivadas con otros índices de daño. Además, es necesario tener mayor claridad en cuanto a los verdaderos niveles de ductilidad y resistencia lateral que son capaces de desarrollar las estructuras durante su respuesta dinámica a una excitación sísmica severa. Por tanto, aunque es importante calibrar varios parámetros involucrados en la determinación de la resistencia lateral de diseño de las estructuras desplantadas en el Distrito Federal antes de hacer recomendaciones especificas para modificar las Normas Técnicas Complementarias para Diseño por Sismo, el estudio presentado aquí permite: A) Observar bajo qué condiciones de suelo y para qué tipo de estructuras es de importancia considerar explícitamente las demandas acumuladas de deformación plástica, y B) Identificar la necesidad de incorporar el efecto de las demandas acumuladas de deformación plástica en el planteamiento de la resistencia lateral de diseño para las estructuras desplantadas en la Zona del Lago del Distrito Federal.

\section{AGRADECIMIENTOS}

Se agradecen los valiosos comentarios y sugerencias de los doctores Luís Eduardo Pérez Rocha y Jorge Ruiz García. El primer autor agradece a CONACYT la beca otorgada durante los estudios de posgrado y dentro del programa de Repatriación, así como el apoyo de la Universidad Autónoma de Sinaloa. El primer autor además agradece a ReLUIS (Rete dei Laboratori Universitari di Ingegneria Sismica) del programa de investigación fundado por el Departamento de Protección Civil en Italia. El segundo autor agradece el apoyo de la Universidad Autónoma Metropolitana. Esta investigación se realizó en su mayor parte dentro del proyecto DGAPA-UNAM 108708. El tercer autor agradece al Instituto de Ingeniería de la UNAM por las facilidades brindadas durante el programa de Verano de la Investigación Científica- 2007.

\section{REFERENCIAS}

Anderson, J C, E Miranda y V V Bertero (1991), "Evaluation of the seismic performance of a 30-story building", Reporte UCB/EERC-91/16, Universidad de California, Berkeley.

Arias, A (1970), "A measure of earthquake intensity”, Seismic Design for Nuclear Power Plants, edited by R. J. Hansen, MIT Press, Cambridge, MA, pp. 438-483.

Arroyo, D y A Terán-Gilmore (2002), "Strength reductions factors to account for low cycle fatigue", Seventh US Conference on Earthquake Engineering, Boston, USA, (CD-ROM).

Arroyo, D y M Ordaz (2007), "Hysteretic energy demands for SDOF systems subjected to narrow band earthquake ground motions. Applications to the lake bed zone of Mexico City", Journal of Earthquake Engineering, Vol. 11, pp. 147-165.

Avilés, J y L E Pérez-Rocha (2007), "Damage analysis of structures on elastic foundation", Journal of Structural Engineering, ASCE. Vol. 133, No 10, pp. 1453-1461.

Baik, S W, D G Lee y H Krawinkler (1988), "A simplified model for seismic response prediction of steel frame structures”, IX World Conference on Earthquake Engineering, Tokio, Japón.

Ballio, G y C A Castiglioni (1994), "An approach to the seismic design of steel structures based on cumulative damage criteria", Earthquake Engineering and Structural Dynamics, Vol. 23, pp. 969986.

Bazán, E, I Díaz, J Bielak y N Bazán (1992), "Probabilistic seismic response of inelastic building foundation systems", Memorias, 10th World Conference on Earthquake Engineering, Madrid, España. 
Bertero, V V (1997), "Performance-based seismic engineering: A critical review of proposed guidelines", Seismic Design Methodologies for the Next Generation of Codes, Proceedings, pp. 1-31.

Bojórquez, E y S E Ruiz (2004), "Strength reduction factors for the valley of Mexico taking into account low cycle fatigue effects", $13^{\circ}$ World Conference on Earthquake Engineering, paper 516, Vancouver, Canada 2004 (CD-ROM).

Bojórquez, E y J L Rivera (2005), "Espectros con tasa de falla uniforme en S1GL para distintos modelos de comportamiento teóricos (utilizando funciones de degradación)", XV Congreso Nacional de Ingeniería Sísmica, Ciudad de México 2005 (CD-ROM).

Bojórquez, E, I Iervolino, G Manfredi y E Cosenza (2006), "Influence of ground motion duration on degrading SDOF systems", First European Conference on Earthquake Engineering and Seismology, Geneva, Switzerland (CD-ROM).

Bojórquez, E (2007), "Vulnerabilidad sísmica de edificios usando conceptos de energía", Tesis de Doctorado, Universidad Nacional Autónoma de México.

Bojórquez, E, S E Ruiz y A Terán-Gilmore (2008a), "Reliability-based evaluation of steel structures using energy concepts", Engineering Structures, Vol. 30, No. 6, pp. 1745-1759.

Bojórquez, E, A Terán-Gilmore, S E Ruiz y A Reyes-Salazar (2008b), "Evaluation of structural reliability of steel frames considering cumulative damage", The $14^{\text {th }}$ World Conference on Earthquake Engineering, Beijing, China 2008 (CD-ROM).

Boomer, J M, J Magenes, J Hancock y P Penazzo (2004), "The influence of strong-motion duration on the seismic response of masonry structures", Bulletin of Earthquake Engineering 2004, Vol. 2, pp. 126.

Bozorgnia, Y y V V Bertero (2001), "Improved shaking and damage parameters for post-earthquake applications", Proceedings of the SMIP01 Seminar on Utilization of Strong-Motion Data, Los Angeles, California.

Cosenza, E, G Manfredi y R Ramasco (1993), "The use of damage functionals in earthquake engineering: a comparison between different methods", Earthquake Engineering and Structural Dynamics, Vol. 22 , pp. 855-868.

Cosenza, E y G Manfredi (1996), "Seismic design based on low cycle fatigue criteria", Memorias XI Congreso Mundial de Ingeniería Sísmica, Acapulco, Mexico (CD-ROM).

Chai, Y H y K M Romstad (1997), "Correlation between strain-based low-cycle fatigue and energy-based linear damage models", Earthquake Spectra, Vol. 13 No. 2, 191-209.

Chai, Y H (2005), "Incorporating low-cycle fatigue model into duration-dependent inelastic design spectra”, Earthquake Engineering and Structural Dynamics, Vol. 23, pp. 1023-1043.

Chopra, A K, (2001), Dynamics of Structures. Theory and applications to earthquake engineering, Prentice Hall, Second Edition, New Jersey, 844pp.

Chou, C C y C M Uang (2003), "A Procedure to evaluate energy demand for framed structures" Earthquake Engineering and Structural Dynamics, Vol. 32, pp. 229-244.

Espinoza-Johnson, M A y A Terán-Gilmore (2000), "Efecto de la degradación de rigidez en las demandas sísmicas de sistemas simples", Memorias XII Congreso Nacional de Ingeniería Estructural (CDROM). 
Fajfar, P (1992), "Equivalent ductility factors taking into account low-cycle fatigue", Earthquake Engineering and Structural Dynamics, Vol. 21, pp. 837-848.

Hancock, J y J M Bommer (2006), "A state-of-knowledge review of the influence of strong-motion duration on structural damage", Earthquake Spectra, Vol. 22, No. 3, pp. 827-845.

Huerta, B y E Reinoso (2002), "Espectros de energía de movimientos fuertes registrados en México", Revista de Ingeniería Sísmica, SMIS, Vol. 66, pp. 45-72.

Iervolino, I, G Manfredi y E Cosenza (2006), "Ground motion duration effects on nonlinear seismic response", Earthquake Engineering and Structural Dynamics, Vol. 35, pp. 21.38.

Krawinkler, H y M Zohrei (1983), "Cumulative damage in steel structure subjected to earthquake ground motions”, Computer and Structures, Vol. 16 pp. 531-541.

Kunnath, S K, A El-Bahy, A W Taylor y W C Stone (1999), "Cumulative seismic damage in reinforced concrete bridge columns: Benchmarks and low cycle fatigue tests", ACI Structural Journal, Vol. 96, No. 4, pp. 633-643.

Lam, N, J Wilson y G Hutchinson (1998), "The ductility reduction factor in the seismic design of buildings", Earthquake Engineering and Structural Dynamics, Vol. 27, pp. 749-769.

Lee, L H, S W Han y Y H Oh (1999), "Determination of ductility factor considering different hysteretic models", Earthquake Engineering and Structural Dynamics, Vol. 28, pp. 957-977.

Malhotra, P K (2002), "Cyclic-demand spectrum", Earthquake Engineering and Structural Dynamics, Vol. 31, pp. 1441-1457.

Manfredi, G (2001), "Evaluation of seismic energy demand", Earthquake Engineering and Structural Dynamics, Vol. 30 pp. 485-499.

Mehanny, S F y G G Deierlein (2000), "Modeling of assessment of seismic performance of composite frames with reinforced concrete columns and steel beams", Report No. 135, The John A. Blume Earthquake Engineering Center, Stanford University.

Miranda, E y V V Bertero (1989), “The Mexico earthquake of September 19, 1985-performance of lowrise buildings in Mexico City, Earthquake Spectra, Vol. 5, No. 1, 121-143.

Miranda, E (1993), "Site-dependent strength reduction factors", J. Structural. Engineering, Vol. 119, pp. 3503-3519.

Miranda, E y V V Bertero (1994), "Evaluation of strength reduction factors for earthquake-resistant design", Earthquake Spectra, Vol. 10, No. 2, pp. 357-379.

Miranda, E y J Ruiz (1999), "Influencia de la degradación de rigidez en las demandas de desplazamiento lateral de estructuras cimentadas en suelos blandos", Memorias XII Congreso Nacional de Ingeniería Sísmica, Morelia, Michoacán (CD_ROM).

Miranda, E y J Ruiz-García (2002), "Influence of stiffness degradation on strength demands of structures built on soft soil sites", Engineering Structures, Vol. 24, No. 10, pp. 1271-1281.

Muria, D y R González (1995), "Propiedades dinámicas de edificios de la ciudad de México", Revista de Ingeniería Sísmica, SMIS, Vol. 51, pp. 25-45.

Muria, D, L Fuentes y R González (2000), "Uncertainties in the estimation of natural frequencies of buildings in Mexico City", $12^{\text {th }}$ World Conference on Earthquake Engineering, Auckland, Nueva Zelanda (CD-ROM). 
Ordaz, M y L E Pérez (1998), "Estimation of strength-reduction factors for elastoplastic systems: a new approach", Earthquake Engineering and Structural Dynamics., Vol. 27, pp. 889-901.

Ordaz, M, E Miranda y J Avilés (2003), “Propuesta de espectros de diseño por sismo para el DF." Revista Internacional de Ingeniería de Estructuras, editado por Escuela Superior Politécnica del Ejército, 8, 189-208.

Panagiotakos, T B y M N Fardis (2001), "Deformations of reinforced concrete members at yielding and ultimate", ACI Structural. Journal, Vol. 98, No. 2, pp. 135-148.

Park, Y J y A H Ang (1985), "Mechanistic seismic damage model for reinforced concrete", ASCE Journal of Structural Engineering, Vol. 111, No. ST4, pp. 740-757.

Paulay, T (1996), "Seismic design of concrete structures the present need of societies", Memorias XI Congreso Mundial de Ingeniería Sísmica, Acapulco, Mexico (CD-ROM).

Pérez-Rocha, L E y J Avilés (2003), "Evaluación de efectos de interacción en resistencias inelásticos", Revista de Ingeniería Sísmica, SMIS, Vol. 69, pp. 45-71.

Powell, G H y R Allahabadi (1987), "Seismic damage prediction by deterministic methods: concepts and procedures", Earthquake Engineering and Structural Dynamics, Vol. 16, 719-734.

Quiroz-Ramírez, A y A Terán-Gilmore (2008), "Método Empírico de Escalado que Considera los Contenidos de Energía y de Frecuencias de los Movimientos del Terreno", XVI Congreso Nacional de Ingeniería Estructural (CD-ROM).

Reinoso, E (1996), "Algunos resultados recientes sobre el peligro sísmico en la ciudad de México", Revista de Ingeniería Sísmica, SMIS, Vol. 53, pp. 1-24.

Rodríguez, M E (1997), "Una medida de la capacidad destructiva de terremotos", Revista de Ingeniería Sísmica, SMIS, Vol. 55, pp. 37-59.

Rodríguez, M E y R Montes (1998), “Comportamiento sísmico no lineal de edificaciones sobre suelo blando", Revista de Ingeniería Sísmica, SMIS, Vol. 58 pp. 1-20.

Rodríguez, M E y J C Ariztizabal (1999), "Evaluation of a seismic damage parameter", Earthquake Engineering and Structural Dynamics, Vol. 28, pp. 463-477.

Rodríguez, M E y D Padilla. (2008), "A damage index for the seismic analysis of reinforced concrete members", artículo aceptado para Journal of Earthquake Engineering.

Ruiz-Garcia, J y E Miranda (2004), "Inelastic Displacement Ratios for Structures Built on Soft Soil Sites", Journal of Structural Engineering, Vol. 130, No. 12, pp. 2051-2061.

Silva-Olivera, H y O López-Batiz (2001), "Estudio experimental sobre índices de daño en estructuras de concreto reforzado sujetas a cargas laterales", XIII Congreso Nacional de Ingeniería Sísmica, Guadalajara, México (CD_ROM).

Stephens, J E y J T P Yao (1987), "Damage assessment using response measurements", ASCE Journal of Structural Engineering, Vol. 113, No. 4, 787-801.

Tarquis, F y J M Roesset (1988), "Structural response and design spectra for the 1985 Mexico City earthquake", Report No: GD89-1, University of Texas, Austin, TX.

Terán-Gilmore, A (1996), "Performance-based earthquake-resistant design of framed building using energy concepts", Tesis de Doctorado, University of California Berkley.

Terán-Gilmore, A (2001), "Consideraciones del uso de la energía plástica en el diseño sísmico", Revista de ingeniería Sísmica, SMIS, Vol. 65, pp. 81-110. 
Terán-Gilmore, A y M A Espinoza-Johnson (2003), "Resistencia de diseño para sistemas simples que exhiben degradación de rigidez y resistencia", Memorias XIV Congreso Nacional de Ingeniería Sísmica (CD_ROM).

Terán-Gilmore, A y J O Jirsa (2005), "A damage model for practical seismic design that accounts for low cycle fatigue", Earthquake Spectra, Vol. 21, No. 3, pp. 803-832.

Terán-Gilmore, A y J O Jirsa (2007), "Energy demands for seismic design against low-cycle fatigue", Earthquake Engineering and Structural Dynamics, Vol. 36, pp. 383-404.

Terán-Gilmore, A y M A Espinoza (2008), "Diseño por desempeño de estructuras dúctiles de concreto reforzado ubicadas en la zona del lago del Distrito Federal: la resistencia lateral de diseño", Revista de Ingeniería Sísmica, SMIS, Vol.78, pp. 23-46.

Uang, C M (1991), "Establishing $\mathrm{R}$ (or $\mathrm{R}_{\mathrm{w}}$ ) and $\mathrm{C}_{\mathrm{d}}$ factors for Building Seismic Provisions", ASCE Journal of Structural Engineering, Vol. 117, No. 1, 19-28.

Villa-Velázquez, C I y S E Ruiz (2001), "Influencia de la intensidad en las características de los movimientos registrados en el valle de México", Informe elaborado para la Secretaría de Obras y Servicios del D.F., Instituto de Ingeniería, UNAM.

Whittaker, A, G Hart y C Rojahn (1999), "Seismic response modification factors", J. Structural Engineering (ASCE), Vol. 125, No. 4, pp. 438-444.

Williams, M S y R G Sexsmith (1995), "Seismic damage indices for concrete structures: a state of the art review", Earthquake Spectra, Vol. 11, No. 2, pp. 319-349.

Williams, M S y R G Sexsmith (1997), "Seismic assessment of concrete bridges using inelastic damage analysis", Engineering Structures, Vol. 19, No. 3, pp. 208-216. 
Edén Bojórquez Mora, Amador Terán Gilmore, Juan Bojórquez Mora y Sonia E Ruiz Gómez

APÉNDICE A. REGISTROS SÍSMICOS UTILIZADOS (Villa-Velázquez y Ruiz, 2001)

Tabla A.1 Registros sísmicos de la zona I.

\begin{tabular}{|c|c|c|c|c|c|c|}
\hline \multirow{2}{*}{ Registro } & \multirow{2}{*}{ Fecha } & \multirow{2}{*}{ Magnitud } & \multirow{2}{*}{ Nombre de la Estación } & \multicolumn{2}{|c|}{ Coordenadas de la Estación } & \multirow{2}{*}{$\begin{array}{c}\mathrm{Ams} \\
\left(\mathrm{cm} / \mathrm{s}^{2}\right)\end{array}$} \\
\hline & & & & $\begin{array}{c}\text { Latitud } \\
\mathrm{N}\end{array}$ & $\begin{array}{c}\text { Longitud } \\
\text { W }\end{array}$ & \\
\hline 1 & $79-03-14$ & 7.0 & Sismex CU & 19.326 & 99.182 & 12.9 \\
\hline 2 & $79-03-14$ & 7.0 & Sismex Hospital ABC & 19 & 99.205 & 14.3 \\
\hline 3 & $82-06-07$ & 6.9 & ii inst. sísmica & 19.33 & 99.183 & 30.3 \\
\hline 4 & $85-09-19$ & 8.1 & ii inst. sísmica & 19.33 & 99.183 & 32 \\
\hline 5 & $85-09-19$ & 8.1 & ii patio & 19.33 & 99.183 & 36.2 \\
\hline 6 & $85-09-19$ & 8.1 & Mesa vibradora & 19.33 & 99.183 & 34.6 \\
\hline 7 & $85-09-19$ & 8.1 & Tacubaya & 19.403 & 99.194 & 18.2 \\
\hline 8 & $85-09-21$ & 7.6 & ii patio & 19.33 & 99.183 & 13.6 \\
\hline 9 & $85-09-21$ & 7.6 & Mesa vibradora & 19.33 & 99.183 & 13 \\
\hline 10 & $85-09-21$ & 6.9 & Lab. de desarrollo & 19.33 & 99.183 & 17.3 \\
\hline 11 & $85-09-21$ & 6.9 & $\begin{array}{c}\text { Fund. Javier Barros } \\
\text { Sierra }\end{array}$ & 19.299 & 99.21 & 15.5 \\
\hline 12 & $85-09-21$ & 6.9 & Mariscal Tito & 19.425 & 99.19 & 11.4 \\
\hline 13 & $85-09-21$ & 6.9 & San Pedro Atocpan & 19.202 & 99.049 & 15.6 \\
\hline 14 & $85-09-21$ & 6.9 & Tacubaya & 19.403 & 99.194 & 18.3 \\
\hline 15 & $85-09-21$ & 6.9 & Tlalpan & 19.292 & 99.171 & 7.91 \\
\hline 16 & $95-09-14$ & 7.3 & Est. \# 14 Chapultepec & 19.416 & 99.205 & 18.3 \\
\hline 17 & $95-09-14$ & 7.3 & Colinas del Sur & 19.366 & 99.226 & 13.1 \\
\hline 18 & $95-09-14$ & 7.3 & ii patio 4 & 19.33 & 99.183 & 11.6 \\
\hline 19 & $95-09-14$ & 7.3 & $\begin{array}{c}\text { Fund. Javier Barros } \\
\text { Sierra }\end{array}$ & 19.299 & 99.21 & 15.3 \\
\hline 20 & $95-09-14$ & 7.3 & San Pedro Atocpan & 19.202 & 99.049 & 15.4 \\
\hline 21 & $95-09-14$ & 7.3 & Campo libre & 19.351 & 99.186 & 11 \\
\hline 22 & $95-09-14$ & 7.3 & Tacubaya & 19.403 & 99.194 & 12.5 \\
\hline 23 & $95-09-14$ & 7.3 & Tlalpan & 19.292 & 99.171 & 14.4 \\
\hline
\end{tabular}

Tabla A.2 Registros sísmicos de la zona II.

\begin{tabular}{|c|c|c|c|c|c|c|}
\hline \multirow{2}{*}{ Registro } & \multirow{2}{*}{ Fecha } & \multirow{2}{*}{ Magnitud } & \multirow{2}{*}{ Nombre de la Estación } & \multicolumn{2}{|c|}{ Coordenadas de la Estación } & \multirow{2}{*}{$\begin{array}{c}\text { Ams } \\
\left(\mathrm{cm} / \mathrm{s}^{2}\right)\end{array}$} \\
\hline & & & & $\begin{array}{l}\text { Latitud } \\
\mathrm{N}\end{array}$ & $\begin{array}{l}\text { Longitud } \\
\text { W }\end{array}$ & \\
\hline 1 & $81-10-25$ & 7.3 & Sismex Viveros & 19.358 & 99.171 & 15.6 \\
\hline 2 & $85-09-19$ & 8.1 & Sismex Viveros & 19.358 & 99.171 & 55.2 \\
\hline 3 & $89-04-25$ & 6.9 & Angel Urraza & 19.383 & 99.168 & 25.1 \\
\hline 4 & $89-04-25$ & 6.9 & Deportivo Reynoso & 19.501 & 99.183 & 24 \\
\hline 5 & $89-04-25$ & 6.9 & Xotepingo & 19.332 & 99.144 & 39.1 \\
\hline 6 & $89-04-25$ & 6.9 & Escandón & 19.402 & 99.177 & 20.3 \\
\hline 7 & $89-04-25$ & 6.9 & Granjas & 19.475 & 99.18 & 45.6 \\
\hline 8 & $89-04-25$ & 6.9 & Mariano Escobedo & 19.438 & 99.182 & 22.4 \\
\hline 9 & $95-09-14$ & 7.3 & Alberca olímpica & 19.359 & 99.154 & 32.4 \\
\hline 10 & $95-09-14$ & 7.3 & Angel Urraza & 19.383 & 99.168 & 20.8 \\
\hline 11 & $95-09-14$ & 7.3 & Est. \# 6 Coyoacán & 19.348 & 99.169 & 17.8 \\
\hline 12 & $95-09-14$ & 7.3 & Xotepingo & 19.332 & 99.144 & 20.5 \\
\hline 13 & $95-09-14$ & 7.3 & Escandón & 19.402 & 99.177 & 22.9 \\
\hline 14 & $95-09-14$ & 7.3 & Est. \# 15 IMP & 19.488 & 99.149 & 28.3 \\
\hline 15 & $95-09-14$ & 7.3 & Mariano Escobedo & 19.438 & 99.182 & 26.7 \\
\hline 16 & $95-09-14$ & 7.3 & UAM Azcapotzalco & 19.52 & 99.19 & 21.8 \\
\hline 17 & $97-01-11$ & 6.9 & Alberca olímpica & 19.359 & 99.154 & 11.6 \\
\hline 18 & $97-01-11$ & 6.9 & Angel Urraza & 19.383 & 99.168 & 8.87 \\
\hline 19 & $97-01-11$ & 6.9 & Xotepingo & 19.332 & 99.144 & 21.8 \\
\hline 20 & $97-01-11$ & 6.9 & Granjas & 19.475 & 99.18 & 14.1 \\
\hline 21 & $97-01-11$ & 6.9 & Est. \# 15 IMP & 19.488 & 99.149 & 20.3 \\
\hline 22 & $97-01-11$ & 6.9 & Mariano Escobedo & 19.438 & 99.182 & 10.6 \\
\hline
\end{tabular}


Consideración explícita del daño acumulado en el diseño sísmico de estructuras a través de factores de reducción ...

Tabla A.3 Registros sísmicos de la zona IIIa.

\begin{tabular}{|c|c|c|c|c|c|c|}
\hline \multirow{2}{*}{ Registro } & \multirow{2}{*}{ Fecha } & \multirow{2}{*}{ Magnitud } & \multirow{2}{*}{ Nombre de la Estación } & \multicolumn{2}{|c|}{ Coordenadas de la Estación } & \multirow{2}{*}{$\begin{array}{c}\text { Ams } \\
\left(\mathrm{cm} / \mathrm{s}^{2}\right)\end{array}$} \\
\hline & & & & $\begin{array}{c}\text { Latitud } \\
\mathrm{N}\end{array}$ & $\begin{array}{c}\text { Longitud } \\
\mathrm{W}\end{array}$ & \\
\hline 1 & $89-04-25$ & 6.9 & Roma & 19.405 & 99.166 & 48.8 \\
\hline 2 & $89-04-25$ & 6.9 & Ibero & 19.345 & 99.13 & 25.5 \\
\hline 3 & $89-04-25$ & 6.9 & Jardines de Coyoacán & 19.313 & 99.127 & 34.4 \\
\hline 4 & $89-04-25$ & 6.9 & Lindavista & 19.493 & 99.128 & 30.3 \\
\hline 5 & $89-04-25$ & 6.9 & Miramontes & 19.283 & 99.125 & 36.4 \\
\hline 6 & $89-04-25$ & 6.9 & San Simón & 19.375 & 99.148 & 39.6 \\
\hline 7 & $89-04-25$ & 6.9 & Unidad Colonia IMSS & 19.434 & 99.165 & 52.2 \\
\hline 8 & $95-09-14$ & 7.3 & Culhuacán & 19.33 & 99.125 & 30.8 \\
\hline 9 & $95-09-14$ & 7.3 & Ibero & 19.345 & 99.13 & 31.2 \\
\hline 10 & $95-09-14$ & 7.3 & Jardines de Coyoacán & 19.313 & 99.127 & 30.2 \\
\hline 11 & $95-09-14$ & 7.3 & Miramontes & 19.283 & 99.125 & 27.6 \\
\hline 12 & $97-01-11$ & 6.9 & Culhuacán & 19.33 & 99.125 & 12.4 \\
\hline 13 & $97-01-11$ & 6.9 & Roma & 19.405 & 99.166 & 17.6 \\
\hline 14 & $97-01-11$ & 6.9 & Jardines de Coyoacán & 19.313 & 99.127 & 24.1 \\
\hline 15 & $97-01-11$ & 6.9 & Miramontes & 19.283 & 99.125 & 26.2 \\
\hline 16 & $97-01-11$ & 6.9 & San Simón & 19.375 & 99.148 & 17.5 \\
\hline
\end{tabular}

Tabla A.4 Registros sísmicos de la zona IIIb.

\begin{tabular}{|c|c|c|c|c|c|c|}
\hline \multirow{2}{*}{ Registro } & \multirow{2}{*}{ Fecha } & \multirow{2}{*}{ Magnitud } & \multirow{2}{*}{ Nombre de la Estación } & \multicolumn{2}{|c|}{$\begin{array}{l}\text { Coordenadas de la } \\
\text { Estación }\end{array}$} & \multirow{2}{*}{$\begin{array}{c}\text { Ams } \\
\left(\mathrm{cm} / \mathrm{s}^{2}\right)\end{array}$} \\
\hline & & & & $\begin{array}{l}\text { Latitud } \\
\mathrm{N}\end{array}$ & $\begin{array}{l}\text { Longitud } \\
\text { W }\end{array}$ & \\
\hline 1 & $85-09-19$ & 8.1 & SCT & 19.292 & 99.147 & 178 \\
\hline 2 & $85-09-21$ & 7.6 & Tlahuac deportivo & 19.293 & 99.035 & 48.7 \\
\hline 3 & $89-04-25$ & 6.9 & Alameda & 19.436 & 99.145 & 45 \\
\hline 4 & $89-04-25$ & 6.9 & Cibeles & 19.419 & 99.165 & 62.2 \\
\hline 5 & $89-04-25$ & 6.9 & CU Juárez & 19.41 & 99.157 & 49.1 \\
\hline 6 & $89-04-25$ & 6.9 & Garibaldi & 19.439 & 99.14 & 68 \\
\hline 7 & $89-04-25$ & 6.9 & $\mathrm{SCT}$ & 19.393 & 99.147 & 44.9 \\
\hline 8 & $89-04-25$ & 6.9 & Tlatelolco & 19.45 & 99.134 & 52.9 \\
\hline 9 & $89-04-25$ & 6.9 & Tlatelolco & 19.436 & 99.143 & 39.3 \\
\hline 10 & $95-09-14$ & 7.3 & Alameda & 19.436 & 99.145 & 39.1 \\
\hline 11 & $95-09-14$ & 7.3 & CU Juárez & 19.41 & 99.157 & 27.9 \\
\hline 12 & $95-09-14$ & 7.3 & Centro urbano Presidente Juárez & 19.41 & 99.157 & 27.7 \\
\hline 13 & $95-09-14$ & 7.3 & Córdoba & 19.422 & 99.159 & 43.3 \\
\hline 14 & $95-09-14$ & 7.3 & Edif. Jalapa & 19.423 & 99.159 & 29.6 \\
\hline 15 & $95-09-14$ & 7.3 & Garibaldi & 19.439 & 99.14 & 30.1 \\
\hline 16 & $95-09-14$ & 7.3 & Liconsa & 19.306 & 98.963 & 49.5 \\
\hline 17 & $95-09-14$ & 7.3 & Plutarco Elías Calles & 19.39 & 99.132 & 33.5 \\
\hline 18 & $95-09-14$ & 7.3 & Rodolfo Menéndez & 19.436 & 99.128 & 22.3 \\
\hline 19 & $95-09-14$ & 7.3 & Sector Popular & 19.366 & 99.119 & 34.3 \\
\hline 20 & $95-09-14$ & 7.3 & Tlatelolco & 19.45 & 99.134 & 27.5 \\
\hline 21 & $95-09-14$ & 7.3 & Tlatelolco & 19.436 & 99.143 & 27.2 \\
\hline 22 & $95-10-09$ & 7.5 & Cibeles & 19.419 & 99.165 & 14.4 \\
\hline 23 & $95-10-09$ & 7.5 & Centro urbano Presidente Juárez & 19.41 & 99.157 & 15.7 \\
\hline 24 & $95-10-09$ & 7.5 & Edif. Jalapa & 19.423 & 99.159 & 11.4 \\
\hline 25 & $95-10-09$ & 7.5 & Garibaldi & 19.439 & 99.14 & 11 \\
\hline 26 & $95-10-09$ & 7.5 & Liverpool & 19.426 & 99.157 & 17.6 \\
\hline 27 & $95-10-09$ & 7.5 & Plutarco Elías Calles & 19.39 & 99.132 & 19.2 \\
\hline 28 & $95-10-09$ & 7.5 & Rodolfo Menéndez & 19.436 & 99.128 & 10.6 \\
\hline 29 & $95-10-09$ & 7.5 & Sector Popular & 19.366 & 99.119 & 13.7 \\
\hline 30 & $97-01-11$ & 6.9 & CU Juárez & 19.41 & 99.157 & 16.2 \\
\hline 31 & $97-01-11$ & 6.9 & Centro urbano Presidente Juárez & 19.41 & 99.157 & 16.3 \\
\hline 32 & $97-01-11$ & 6.9 & Garibaldi & 19.439 & 99.14 & 18.7 \\
\hline 33 & $97-01-11$ & 6.9 & Liverpool & 19.426 & 99.157 & 22.2 \\
\hline 34 & $97-01-11$ & 6.9 & Sector Popular & 19.366 & 99.119 & 16 \\
\hline 35 & $97-01-11$ & 6.9 & Tlatelolco & 19.45 & 99.134 & 13.4 \\
\hline
\end{tabular}

61 
Edén Bojórquez Mora, Amador Terán Gilmore, Juan Bojórquez Mora y Sonia E Ruiz Gómez

Tabla A.5 Registros sísmicos de la zona IIIc.

\begin{tabular}{|c|c|c|c|c|c|c|}
\hline \multirow{2}{*}{ Registro } & \multirow{2}{*}{ Fecha } & \multirow{2}{*}{ Magnitud } & \multirow{2}{*}{ Nombre de la Estación } & \multicolumn{2}{|c|}{ Coordenadas de la Estación } & \multirow{2}{*}{$\begin{array}{c}\text { Ams } \\
\left(\mathrm{cm} / \mathrm{s}^{2}\right)\end{array}$} \\
\hline & & & & $\begin{array}{c}\text { Latitud } \\
\mathrm{N}\end{array}$ & $\begin{array}{l}\text { Longitud } \\
\text { W }\end{array}$ & \\
\hline 1 & $89-04-25$ & 6.9 & Buenos Aires & 19.41 & 99.145 & 61.9 \\
\hline 2 & $89-04-25$ & 6.9 & Candelaria & 19.426 & 99.118 & 48.7 \\
\hline 3 & $89-04-25$ & 6.9 & Prepa \# 7 La Viga & 19.419 & 99.126 & 46.7 \\
\hline 4 & $89-04-25$ & 6.9 & Jamaica & 19.405 & 99.125 & 36.4 \\
\hline 5 & $89-04-25$ & 6.9 & Villa del Mar & 19.381 & 99.125 & 44.2 \\
\hline 6 & $89-04-25$ & 6.9 & Xochipilli & 19.42 & 99.135 & 52.9 \\
\hline 7 & $95-09-14$ & 7.3 & Apatlaco & 19.381 & 99.107 & 51.6 \\
\hline 8 & $95-09-14$ & 7.3 & Candelaria & 19.426 & 99.118 & 31.4 \\
\hline 9 & $95-09-14$ & 7.3 & Central de abastos oficina & 19.372 & 99.096 & 36.9 \\
\hline 10 & $95-09-14$ & 7.3 & Jamaica & 19.405 & 99.125 & 30.8 \\
\hline 11 & $95-09-14$ & 7.3 & Meyehualco & 19.346 & 99.043 & 50.4 \\
\hline 12 & $95-10-09$ & 7.5 & Apatlaco & 19.381 & 99.107 & 15.8 \\
\hline 13 & $95-10-09$ & 7.5 & Buenos Aires & 19.41 & 99.145 & 22.7 \\
\hline 14 & $95-10-09$ & 7.5 & Candelaria & 19.426 & 99.118 & 20.9 \\
\hline 15 & $95-10-09$ & 7.5 & Jamaica & 19.405 & 99.125 & 13.3 \\
\hline 16 & $95-10-09$ & 7.5 & Xochipilli & 19.42 & 99.135 & 23.5 \\
\hline
\end{tabular}

Tabla A.6 Registros sísmicos de la zona IIId.

\begin{tabular}{|c|c|c|c|c|c|c|}
\hline \multirow{2}{*}{ Registro } & \multirow{2}{*}{ Fecha } & \multirow{2}{*}{ Magnitud } & \multirow{2}{*}{ Nombre de la Estación } & \multicolumn{2}{|c|}{ Coordenadas de la Estación } & \multirow{2}{*}{$\begin{array}{c}\text { Ams } \\
\left(\mathrm{cm} / \mathrm{s}^{2}\right)\end{array}$} \\
\hline & & & & $\begin{array}{c}\text { Latitud } \\
\mathrm{N}\end{array}$ & $\begin{array}{c}\text { Longitud } \\
\text { W }\end{array}$ & \\
\hline 1 & $89-04-25$ & 6.9 & Cetis 57 & 19.386 & 99.054 & 21 \\
\hline 2 & $89-04-25$ & 6.9 & Deportivo Moctezuma & 19.431 & 99.096 & 28.8 \\
\hline 3 & $89-04-25$ & 6.9 & Palacio de los deportes & 19.406 & 99.1 & 22.4 \\
\hline 4 & $95-09-14$ & 7.3 & Deportivo Moctezuma & 19.431 & 99.096 & 26.6 \\
\hline 5 & $95-09-14$ & 7.3 & Palacio de los deportes & 19.406 & 99.1 & 29.2 \\
\hline 6 & $95-10-09$ & 7.5 & Aragón & 19.481 & 99.076 & 17.5 \\
\hline 7 & $95-10-09$ & 7.5 & Autódromo & 19.392 & 99.087 & 15 \\
\hline 8 & $95-10-09$ & 7.5 & Deportivo Moctezuma & 19.431 & 99.096 & 17.5 \\
\hline 9 & $95-10-09$ & 7.5 & Hangares & 19.418 & 99.079 & 16.2 \\
\hline 10 & $95-10-09$ & 7.5 & Palacio de los deportes & 19.406 & 99.1 & 17.7 \\
\hline 11 & $95-10-09$ & 7.5 & Est. \# 7 Tlacotal & 19.397 & 99.105 & 12.1 \\
\hline 12 & $95-10-09$ & 7.5 & Est. \# 8 Zaragoza & 19.419 & 99.088 & 16.9 \\
\hline 13 & $97-01-11$ & 6.9 & Autódromo & 19.392 & 99.087 & 22.3 \\
\hline 14 & $97-01-11$ & 6.9 & Cetis 57 & 19.386 & 99.054 & 14 \\
\hline 15 & $97-01-11$ & 6.9 & Palacio de los deportes & 19.406 & 99.1 & 20.8 \\
\hline 16 & $97-01-11$ & 6.9 & Est. \# 8 Zaragoza & 19.419 & 99.088 & 25.6 \\
\hline
\end{tabular}

